\title{
Relief of tumor hypoxia unleashes the tumoricidal potential of neutrophils
}

\author{
Karim Mahiddine, ${ }^{1}$ Adam Blaisdell, ${ }^{2}$ Stephany Ma, ${ }^{1}$ Amandine Créquer-Grandhomme, ${ }^{2}$ Clifford A. Lowell, ${ }^{1,3,4}$ \\ and Adrian Erlebacher ${ }^{1,3,4,5}$
}

'Department of Laboratory Medicine, UCSF, San Francisco, California, USA. ${ }^{2}$ Department of Pathology, NYU School of Medicine, New York, New York, USA. ${ }^{3}$ Biomedical Sciences Program, ${ }^{4}$ ImmunoX Program, and ${ }^{5}$ Center for Reproductive Sciences, UCSF, San Francisco, California, USA.

\begin{abstract}
Polymorphonuclear neutrophils (PMNs) are increasingly recognized to influence solid tumor development, but why their effects are so context dependent and even frequently divergent remains poorly understood. Using an autochthonous mouse model of uterine cancer and the administration of respiratory hyperoxia as a means to improve tumor oxygenation, we provide in vivo evidence that hypoxia is a potent determinant of tumor-associated PMN phenotypes and direct PMN-tumor cell interactions. Upon relief of tumor hypoxia, PMNs were recruited less intensely to the tumorbearing uterus, but the recruited cells much more effectively killed tumor cells, an activity our data moreover suggested was mediated via their production of NADPH oxidase-derived reactive oxygen species and MMP-9. Simultaneously, their ability to promote tumor cell proliferation, which appeared to be mediated via their production of neutrophil elastase, was rendered less effective. Relieving tumor hypoxia thus greatly improved net PMN-dependent tumor control, leading to a massive reduction in tumor burden. Remarkably, this outcome was T cell independent. Together, these findings identify key hypoxia-regulated molecular mechanisms through which PMNs directly induce tumor cell death and proliferation in vivo and suggest that the contrasting properties of PMNs in different tumor settings may in part reflect the effects of hypoxia on direct PMN-tumor cell interactions.
\end{abstract}

\section{Introduction}

Polymorphonuclear neutrophils (PMNs) are thought to play an important, if controversial, role in cancer (reviewed in refs. 1, 2). Elevated blood PMN numbers are associated with poor prognosis in many human cancers, and results from mouse models have suggested that this association variously reflects the ability of PMNs to promote tumor cell proliferation, tumor angiogenesis, metastasis, and the establishment of immunosuppressive tumor microenvironments. Although less well understood, PMNs may also oppose tumorigenesis under certain circumstances (reviewed in ref. 3). For example, PMNs are thought to inhibit the early stages of lung cancer by fostering antitumor $\mathrm{T}$ cell responses (4). A second example comes from our work on endometrioid endometrial adenocarcinoma, the most prevalent gynecological malignancy. Using a mouse model of this cancer, we were able to demonstrate PMN-mediated inhibition of tumor growth that, in this case, occurred in a T cell-independent fashion (5). Our analysis of The Cancer Genome Atlas (TCGA) database, moreover, revealed that patients with endometrioid endometrial cancer survived longer if their primary tumors displayed high PMN transcriptional signatures. Other human cancers, including invasive ductal carcinoma of the breast, low-grade glioma, and colorectal cancer, showed similar positive correlations, even though PMN signatures predict-

Conflict of interest: The authors have declared that no conflict of interest exists. Copyright: (5) 2020, American Society for Clinical Investigation.

Submitted: June 10, 2019; Accepted: October 8, 2019; Published: December 3, 2019.

Reference information: J Clin Invest. 2020;130(1):389-403.

https://doi.org/10.1172/JCl130952. ed poor patient survival when all solid tumor types were considered in aggregate (5). Although controversial, several immunohistochemical studies have also uncovered a correlation between the presence of tumor-associated PMNs and improved patient outcomes in colorectal cancer, and a similar correlation might exist with certain types of lung cancers (2).

The most likely explanation for these divergent associations is that PMNs assume different phenotypes within different tumor microenvironments $(1,2,6)$. For example, early studies suggested that tumor-associated PMNs can assume either a proinflammatory N1 phenotype or an immunosuppressive, tumor-promoting N2 phenotype (7). In recent work, it has been found that a SiglecFi PMN subset promotes tumor growth in a mouse model of lung cancer (8) and that PMNs expressing class II HLA molecules accumulate in human lung cancer specimens (9). In these and other cases, however, the key characteristics of the tumor microenvironment that determine resident PMN phenotypes remain unclear. Elucidating the contributions of PMNs to tumorigenesis is further complicated by the likelihood that PMN-derived products, which are frequently cytotoxic for tumor cells in vitro $(3,10,11)$, may each have their own complex and context-dependent effects in vivo. For example, neutrophil elastase (NE) and MMP-9 are both able to cause epithelial cell death, as evidenced by their contributions to epidermal destruction in bullous pemphigoid disease (12, 13), but NE can also directly promote tumor cell proliferation in lung cancer (14), while MMP-9 promotes tumor angiogenesis (15). Similarly, PMN-derived reactive oxygen species (ROS) contribute to epithelial damage in inflammatory bowel disease (16) and can restrain malignant processes at pulmonary metastatic sites (17), 
but are also thought to contribute to the generation of immunosuppressive tumor microenvironments (18).

Hypoxia, a universal if variable feature of all solid tumors, is well known to modulate PMN phenotypes (reviewed in refs. 19, 20). When studied outside the context of cancer, hypoxia has been shown to augment PMN lifespan (21) and to promote PMN degranulation and protease release (22), effects that are likely in part mediated via activation of the hypoxia-sensing transcription factors HIF-1 $\alpha$ and HIF- $2 \alpha(21,23,24)$. On the other hand, hypoxia reduces the capacity of PMNs to produce ROS (25). Although less well studied, hypoxia in the tumor microenvironment is thought to promote the recruitment and $\mathrm{T}$ cell-suppressive activity of polymorphonuclear (i.e., granulocytic) myeloid-derived suppressor cells (PMN-MDSCs) (26), cells considered to be the product of a deranged PMN differentiation program $(1,2,27)$. Importantly, much of the work on the effects of hypoxia on PMNs and PMN-MDSCs has been performed on isolated cells in vitro or through the use of mice with myeloid cell-targeted genetic disruptions of HIF pathway components $(20-26,28,29)$. Since altered $\mathrm{O}_{2}$ levels within tissues are expected to have both direct and indirect effects on any given cell type, however, the net effect of hypoxia on PMNs within the overall context of the tumor microenvironment remains incompletely understood. In addition to being fundamental to our understanding of how PMNs contribute to tumor development, this question has gained even greater salience given the emerging possibility that hypoxia relief could be used an adjunct modality in cancer immunotherapy $(30,31)$. Indeed, recent work in mice has suggested that therapeutic relief of tumor hypoxia is able to augment antitumor $\mathrm{T}$ cell responses both following immune checkpoint blockade and when applied as the sole manipulation $(32,33)$. In the context of checkpoint blockade, the effect has in turn been linked to less MDSC accumulation and less T cell-suppressive MDSC phenotypes (33). Whether altered $\mathrm{O}_{2}$ levels might also affect the ability of PMNs to act as direct antitumor effectors, however, has not yet been addressed.

Here, we provide in vivo evidence that one of the aggregate, top-down effects of tumor hypoxia in vivo is to limit the ability of PMNs to directly combat tumorigenesis, independently of $\mathrm{T}$ cells. Moreover, we identify specific PMN effector functions through which hypoxia exerts its effects in vivo, namely the induction of tumor cell death by ROS and MMP-9, which is inhibited by hypoxia, and the induction of tumor cell proliferation by NE, which is promoted by hypoxia. This work employs use of respiratory hyperoxia as a means to directly manipulate tumor hypoxia and builds on our aforementioned mouse model of endometrioid endometrial adenocarcinoma. This model is generated by expressing Cre recombinase from the progesterone receptor (PR) promoter to drive uterus-specific deletion of Pten, the tumor suppressor gene whose loss drives this disease in humans $(5,34,35)$. In such PRPL (PR-Cre $\left[\mathrm{Pgr}^{\left.-\mathrm{Cre}^{+/}\right]} \mathrm{Pten}^{\mathrm{fl} / \mathrm{fl}}\right.$ ) mice, uterine epithelial hyperplasia is evident by 2 weeks of age, and progresses synchronously throughout the uterus to carcinoma in situ by approximately 4 weeks of age. Previously, we showed that PMNs massively infiltrate PRPL tumors, become activated via a MyD88-dependent pathway, and then oppose tumor growth as evidenced in part by the markedly increased tumor burden apparent when we depleted the mice of PMNs, either via antibody-mediated neutralization of the growth factor G-CSF, or via genetic disruption of Csf3r, which encodes its receptor (5). Accordingly, the survival of PRPL-Csf $3 r^{--}$mice was also shorter than that of PRPL mice. Through the analysis of 4-week-old mice, we moreover found the antitumor effects of PMNs to be lymphocyte independent, as tumor burden was unaffected when the mice were additionally rendered deficient in either Rag2 or Rag2/Il2rg. Instead, these effects were attributable to an ability of PMNs to induce the detachment of live PRPL tumor cells from their basement membrane, leading to their death secondarily within the uterine lumen. Importantly, this previous study also linked tumor-associated PMN trafficking to tumor hypoxia, since PMNs began infiltrating PRPL tumors when they first became hypoxic and then aggregated in areas of severe tumor hypoxia. Our analysis of the TCGA database moreover supported an association between hypoxia and PMN infiltration in human endometrial cancer (5). We now show that hypoxia indeed promotes PMN recruitment to PRPL tumors, but then alters their phenotype in situ to limit their ability to directly inhibit tumor growth. Upon therapeutic relief of tumor hypoxia, PMN-dependent tumor control was greatly enhanced despite a reduction in PMN infiltration, and this occurred in a $\mathrm{T}$ cell-independent fashion. These findings bear on our understanding of the contribution of PMNs toward tumorigenesis and suggest that PMNs might play a greater role than previously anticipated as direct antitumor effectors in the context of therapeutic hypoxia relief.

\section{Results}

Relief of tumor hypoxia reduces PMN infiltration into PRPL tumors but improves PMN-dependent tumor control. To directly assess the role of hypoxia in PMN-tumor cell interactions, we housed PRPL mice in a $60 \% \mathrm{O}_{2}$ environmental chamber. This high level of respiratory $\mathrm{O}_{2}$ exposure is thought to increase oxygen delivery to tissues by increasing the partial pressure of $\mathrm{O}_{2}$ dissolved in arterial blood (36) and was previously shown to be able to improve the oxygenation of the non-tumor-bearing mouse uterus (37). We chose to house the mice in the chamber starting on postnatal day 18 (P18), because this time point corresponded to when the tumors first started accumulating PMNs and showing signs of hypoxia (5), and to sacrifice them 10 days later on P28, because P28 was the primary terminal end point we employed in our previous study (5). As controls, "normoxia" mice were maintained at ambient $\mathrm{O}_{2}$ for the same time period. Consistent with results from other species (38), 10 days of hyperoxia exposure did not induce lung inflammation (Supplemental Figure 1, A-C; supplemental material available online with this article; https://doi.org/10.1172/JCI130952DS1).

Strikingly, hyperoxia housing reduced the uterine tissue densities (cells/mg tissue) of PMNs by approximately 60\% (Figure $1 \mathrm{~A}$ and Supplemental Figure 1D), without affecting the densities of other uterine leukocyte species (Supplemental Figure 1E). Conversely, blood PMN concentrations were modestly increased following hyperoxia exposure (Figure 1B), but this could not be attributed to an increase in immature PMN release from the bone marrow because blood (as well as uterine) PMNs showed the same Ly6 $\mathrm{G}^{\text {hi }}$ fully mature (39) phenotype under both hyperoxia and normoxia housing conditions (Supplemental Figure 1, $\mathrm{F}-\mathrm{H})$. Rather, we found that hyperoxia housing decreased tumor cell production of CXCL5, a potent PMN chemoattractant and 
A

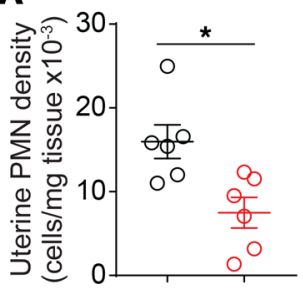

B

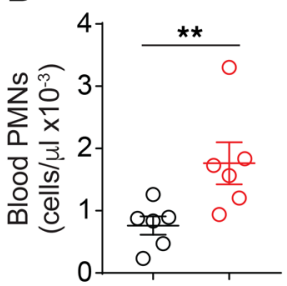

C

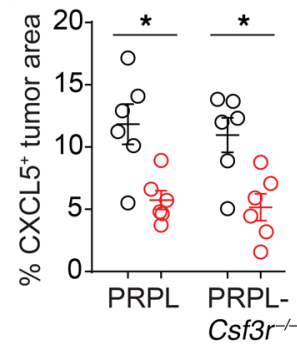

D

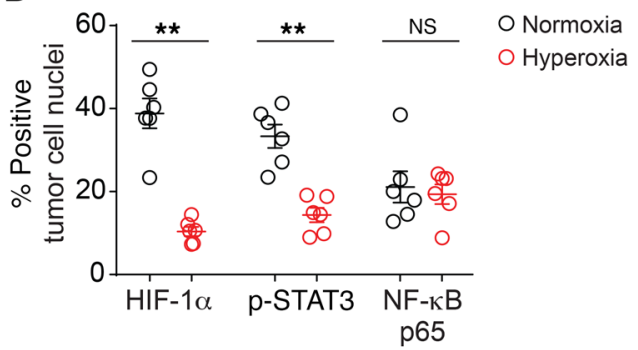

E

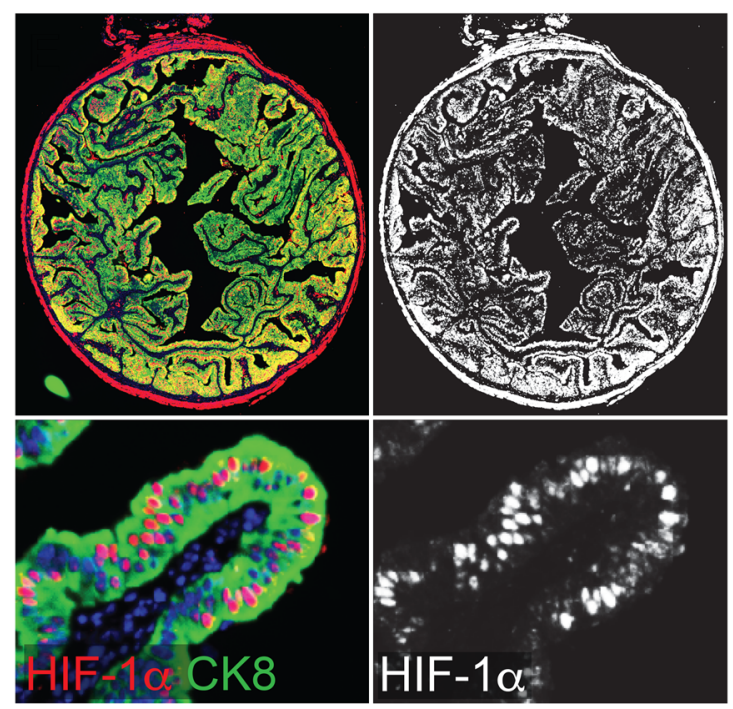

$\mathbf{F}$

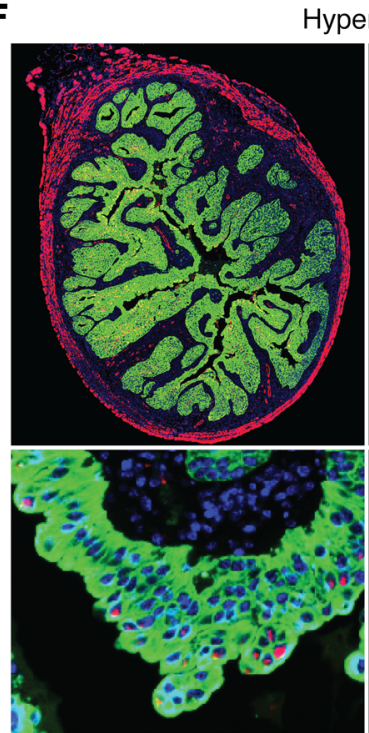

Hyperoxia

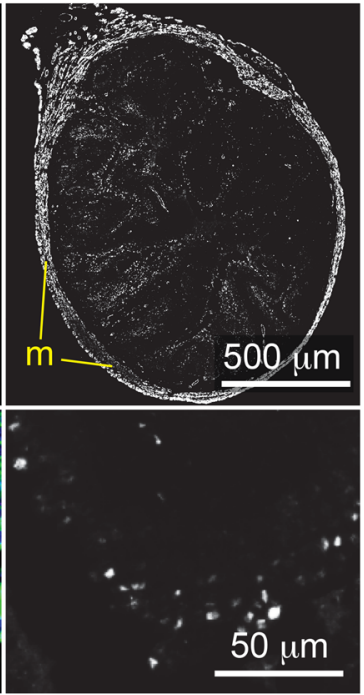

Figure 1. Improved tumor oxygenation reduces PMN recruitment to PRPL tumors. Mice were housed in either ambient $\mathrm{O}_{2}$ (normoxia conditions) or $60 \%$ $\mathrm{O}_{2}$ (hyperoxia conditions) for the last 10 days prior to sacrifice on P28. (A and B) Uterine PMN densities and blood PMN concentrations in PRPL mice, as determined by flow cytometry. Uteri were enzymatically disaggregated prior to analysis. PMNs were identified as CD45+Ly6C ${ }^{\text {hi }}$ cells (see Supplemental Figure 1D for gating). (C) Quantification of CXCL5 expression by tumor cells. The cross-sectional area of tumor cells expressing CXCL5 was determined by immunofluorescence staining of uterine tissue sections and normalized to the cross-sectional area of all tumor cells, as identified by cytokeratin 8 (CK8) costaining. Supplemental Figure 2, A-D, shows representative images. (D) Quantification of nuclear HIF-1 $\alpha$, phospho-STAT3 (p-STAT3), and NF- $\kappa B$ p65 expression by tumor cells in PRPL-Csf3r-1- mice, as determined by immunofluorescence staining. The area of positive staining overlying tumor cell nuclei was normalized to the total tumor cell nuclear area per section. (E and F) Representative HIF-1 $\alpha / C K 8$-stained sections of PPRL-Csf3r-1- mice, with closeups (lower panels) ( $n=6$ mice/group; DAPI counterstain). The staining in the myometrium $(\mathrm{m})$ appeared artifactual as it was not cell associated. Graphs also show the mean $\pm \mathrm{SEM}$. ${ }^{*} P<0.05 ;{ }^{*} P<0.01$ by 2 -tailed Mann-Whitney $U$ test. NS, not significant.

inflammatory marker (Figure 1C and Supplemental Figure 2, A and B). Importantly, our previous study employed PRPL mice deficient in the CXCL1/2/5 receptor CXCR2 to demonstrate an absolute requirement for CXCR2 ligands in recruiting PMNs from the blood to the PRPL uterus, while our decision to focus on CXCL5 was motivated by our prior demonstration that CXCL5 was the CXCR2 ligand most highly induced in PRPL uteri following the onset of tumor hypoxia at approximately 3 weeks of age (5), as well as the existence of CXCL5-specific antibodies suitable for immunofluorescence that enabled us to determine expression levels on a per-tumor-cell basis. Thus, our new observations taken together not only demonstrated that hyperoxia housing could decrease uterine PMN densities in PRPL mice, but also suggested that a major underlying mechanism was reduced PMN recruitment from the blood secondary, at least in part, to decreased CXCL5 production by PRPL tumor cells. The reduction in PMN recruitment in turn likely caused a backup of mature cells in the blood.
Importantly, PMNs themselves can cause tissue hypoxia (40) and are major contributors to tumor inflammation (1), thus creating the potential for feed-forward loops. Accordingly, we also applied respiratory hyperoxia to PRPL-Csf3r- mice, which are markedly deficient in uterine PMNs (ref. 5 and see below), in order to identify which of its intrauterine effects were PMN independent. As with PRPL mice, the tumor cells of hyperoxia-housed PRPLCsf $3 r^{-1-}$ mice expressed less CXCL5 than their normoxia counterparts (Figure 1C and Supplemental Figure 2, C and D). Moreover, they showed much less nuclear accumulation of HIF-1 $\alpha$, a direct marker of hypoxia (Figure 1, D-F), as well as less nuclear accumulation of phospho-STAT3, which we found was in turn required for CXCL5 induction (Figure 1D, Supplemental Figure 2, E and F, and Supplemental Figure 3). In contrast, their level of nuclear NF- $\kappa \mathrm{B}$ $\mathrm{p} 65$, another inflammatory marker, remained unchanged (Figure 1D and Supplemental Figure 2, G and H). Together these results suggested that respiratory hyperoxia improved PRPL tumor oxygenation in a PMN-independent fashion and that the ensuing 

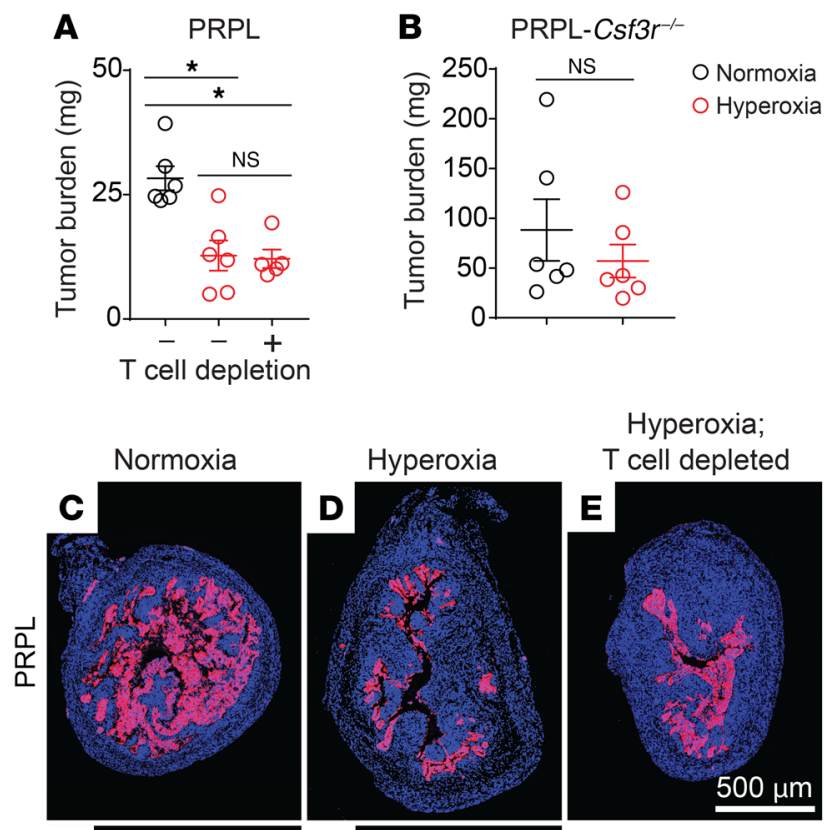

Hyperoxia; T cell depleted
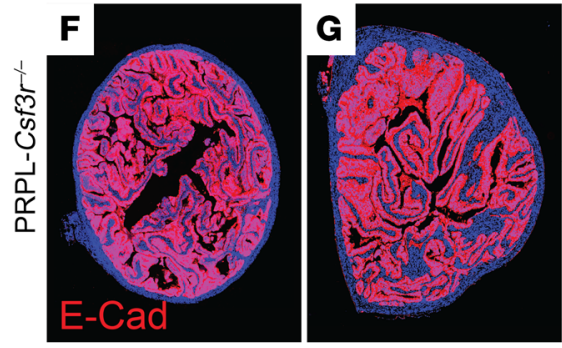

E

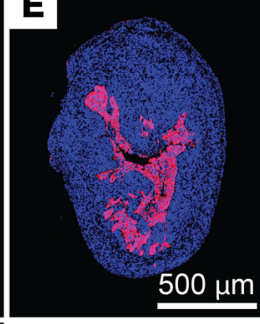

H Pten fitti: normoxia

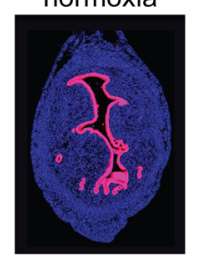

relief of tumor hypoxia had several PMN-independent effects on PRPL tumor cells, including decreased CXCL5 expression that in turn reduced PMN recruitment.

Given these results and our prior evidence that PMNs oppose PRPL tumor growth (5), we were surprised to find that the tumor burden of PRPL mice housed in hyperoxia conditions - calculated from measurements of uterine weights and histological assessments of how much each uterus was composed of tumor cells (Supplemental Figure 4, A and B) - was 2.3-fold lower than the tumor burden of PRPL mice housed in normoxia conditions (Figure 2, A, C, and D; as an additional point of comparison, Figure $2 \mathrm{H}$ shows, to scale, a section from a non-tumor-bearing control Pten $^{f / f l}[$ [PL"] mouse on P28). The reduction in tumor burden was still PMN dependent, however, since hyperoxia housing did not alter the high tumor burden of PRPL-Csf3r/- mice (Figure 2, B, F, and G, and Supplemental Figure 4, A and B). Importantly, these divergent outcomes were not a consequence of differences in tumor burden between PRPL and PRPL-Csf3r/- mice at the time we commenced hyperoxia exposure on P18; rather, tumor burdens on P18 were equivalent (Supplemental Figure 4D), consistent with this time point marking the initial onset of tumor hypoxia and PMN infiltration. Moreover, the reduction in tumor burden in PRPL mice following hyperoxia exposure was $\mathrm{T}$ cell independent since it was also apparent when the mice were concurrently $\mathrm{T}$ cell depleted (Figure 2, A and E, and Supplemental Figure 4, A-C), consistent with our aforementioned observation that 4-week-old PRPL, PRPL-Rag2 ${ }^{-/}$, and PRPL-Rag2 ${ }^{-/-} \mathrm{Il}_{2 g^{-/-}}$mice all have sim-
Figure 2. Relief of tumor hypoxia improves net PMN-dependent tumor control. (A and B) Tumor burden, calculated as the product of uterine weight and percentage cross-sectional area of uterus composed of tumor cells (Supplemental Figure 4, A and B, shows these values). Tumor cells were identified by E-cadherin (E-Cad) immunostaining. The mice were sacrificed on P28; T cells were depleted by administering anti-CD4 and anti-CD8 antibodies on P18, P21, P24, and P27. (C-H) Representative E-Cad-stained sections ( $n=6$ mice/group; DAPI counterstain). H shows, to scale, a section from a non-tumor-bearing control Pten ${ }^{f / / f l}$ ("PL") mouse on $\mathrm{P} 28$ as an additional point of comparison. Graphs also show the mean \pm SEM. ${ }^{*} P<0.05$ by 2 -tailed Mann-Whitney $U$ test. The data in $\mathbf{A}$ were first assessed by the Kruskal-Wallis test $(P<0.01)$ and $P$ values were Bonferroni adjusted for multiple comparisons.

ilar tumor burdens when housed under normoxia conditions (5). Together, these data suggested that hypoxia had a net inhibitory effect on the capacity of PMNs to directly restrain tumor growth, despite its promotion of PMN recruitment.

Tumor hypoxia influences both PMN-induced tumor cell death and PMN-induced tumor cell proliferation. Previously, by comparing normoxia-housed PRPL with PRPL-Csf3r/- mice, we had found that PMNs induced both tumor cell death and tumor cell proliferation (5). The cause of cell death was not the direct induction of tumor cell apoptosis but rather a process we referred to as tumor cell "sloughing" in which PMNs caused live tumor cells to detach from their basement membrane, leaving behind prominent segments of endometrial stromal surfaces "denuded" of their overlying epithelium and thus directly contacting the uterine lumen (e.g., see Figure 3, A and B). Consistent with such a process, the tumor cells in PRPL mice comprising epithelial sheets still attached to the endometrial stroma had lost their basolateral polarization of integrin $\alpha 6 \beta 4$, an integrin that is critical for epithelial cell adhesion to basement membranes. This polarization was still evident in PRPL-Csf $3 r^{-/}$mice (Figure 3, C and D, shows representative staining for the $\alpha 6$ subunit). Following detachment, tumor cell debris admixed with PMNs could be seen within the uterine lumen, with the cells presumably dying by either apoptosis or necrosis. In this prior report, we interpreted the PMN-dependent increase in tumor cell proliferation as a wound-healing response induced secondary to the denudation of the endometrial stroma.

Thus, to gain greater insight into how hypoxia limited the capacity of PMNs to restrain PRPL tumorigenesis, we determined how hyperoxia housing respectively affected tumor cell death and proliferation. Strikingly, hyperoxia housing increased the amount of sloughed tumor and denuded endometrial surface, while it decreased the amount of tumor epithelium with basolateral $\alpha 6$ polarization as well as the rate of tumor cell proliferation, as measured by phospho-histone $\mathrm{H} 3$ (p-H3) immunostaining to identify mitotic cells (Figure 3, E-J, and Supplemental Figure 5, A and B). These changes were not apparent in PRPL-Csf3r-- mice, indicating that they were PMN dependent (Figure 3, G-J, Figure 2, F and G, and Supplemental Figure 5, C and D). Moreover, the changes were unaffected by $\mathrm{T}$ cell depletion (Supplemental Figure 4, E-H), in accord with $\mathrm{T}$ cells not playing a role in the hyperoxia-induced reduction in PRPL tumor burden (Figure 2A), nor were they associated with a change in tumor cell apoptosis among those cells still attached to their basement membrane, as revealed by an immunofluorescence analysis of cleaved caspase-3 (CC-3) expression 

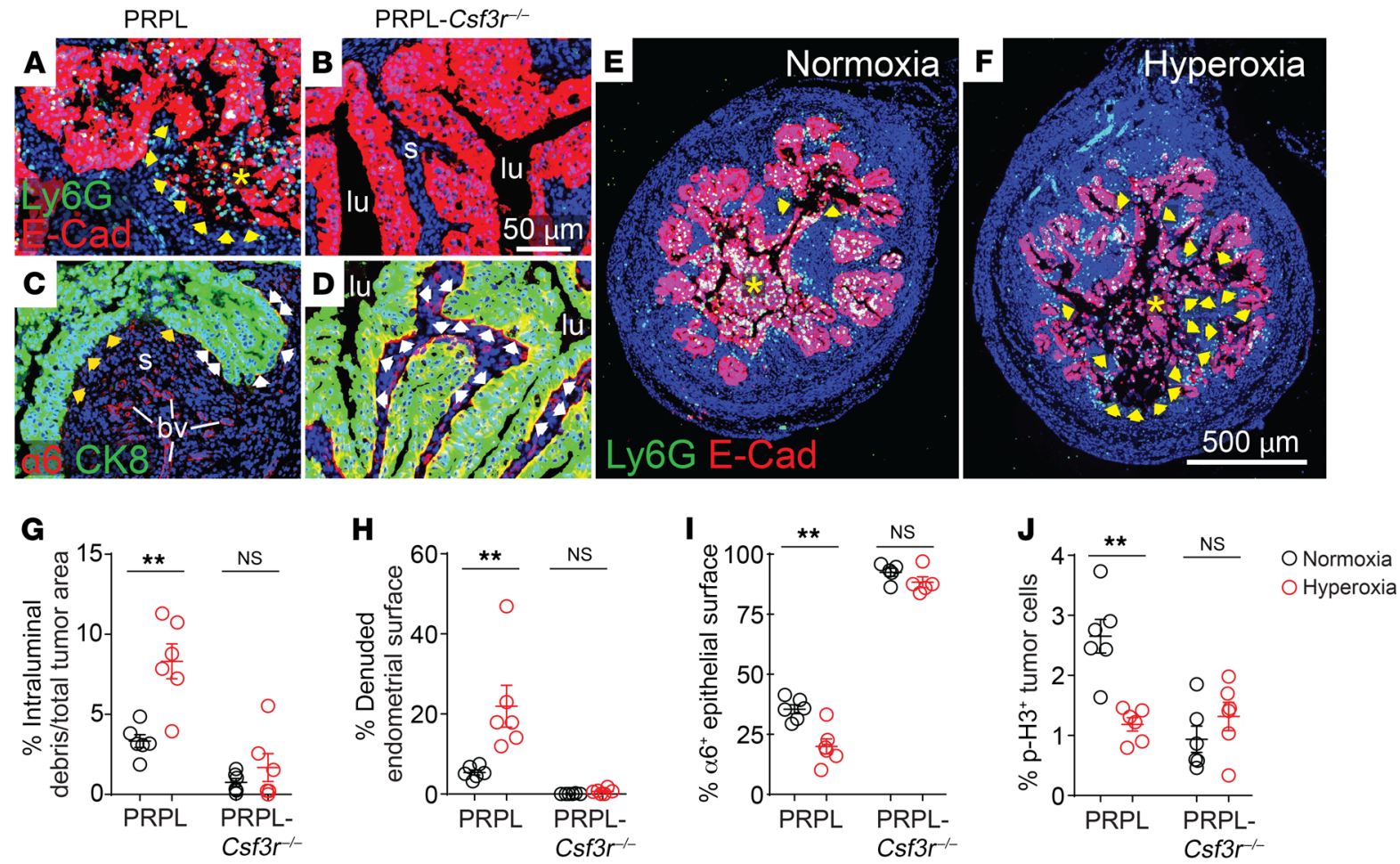

Figure 3. PMNs simultaneously promote and inhibit PRPL tumor growth, with the 2 effects divergently influenced by tumor hypoxia. PRPL (A and C) and PRPL-Csf3r-- (B and D) mice were sacrificed on P28. (A and B) E-Cad/Ly6C double immunostaining to illustrate PMN-dependent tumor cell sloughing and denudation of the uterine stroma (see also ref. 5). Asterisks indicate intraluminal tumor cell debris admixed with PMNs. The arrows demarcate the nearby denuded endometrial surface. s, endometrial stroma; lu, uterine lumen. (C and D) $\alpha 6 / C K 8$ double immunostaining to illustrate PMN-dependent loss of $\alpha 6$ integrin from the basolateral tumor cell membrane (see also ref. 5). In the PRPL mouse (C), note the epithelial/stromal interface completely devoid of $\alpha 6$ staining (yellow arrowheads) and the intermittent $\alpha 6$ staining at other locations along this interface (white arrowheads). Blood vessels (bv) remain $\alpha 6^{+}$. In the PRPL-Csf3r-- mouse (D), $\alpha 6$ staining is largely continuous along the epithelial/stromal interface (see Supplemental Figure 5, A-D, for additional representative images). (E and F) Representative E-Cad-stained uterine sections of PRPL mice housed under normoxia and hyperoxia conditions ( $n=6$ mice/ group) to illustrate sloughed tumor cells (asterisks) and denuded endometrial surfaces (arrows). (G and $\mathbf{H})$ Tumor cell sloughing (percentage intraluminal debris of total tumor area (G) and percentage of denuded endometrial surface $(\mathbf{H})$, determined from E-Cad-stained sections. (I) Percentage of tumor epithelial surface with basolateral $\alpha 6$ integrin staining, determined from $\alpha 6 / C K 8$-stained sections. (J) Tumor cell proliferation, as measured by phospho-histone $\mathrm{H} 3(\mathrm{p}-\mathrm{H} 3)$ immunostaining to identify mitotic cells. Graphs also show the mean $\pm \mathrm{SEM}$. ${ }^{*} P<0.01$ by 2 -tailed Mann-Whitney $U$ test.

(Supplemental Figure 6, A-C). Collectively, these results suggested that improved tumor oxygenation augmented PMN-dependent tumor control via a 2-pronged effect: increased tumor cell death (secondary to increased tumor cell sloughing) and decreased tumor cell proliferation. Moreover, they indicated that the higher rate of tumor cell proliferation in PRPL versus PRPL-Csf3r/- mice under normoxia conditions actually reflected a direct proproliferative effect of PMNs rather than a wound-healing response, since hyperoxia housing decreased tumor cell proliferation while it simultaneously increased tumor cell death.

Individual PMN products have divergent effects on PRPL tumorigenesis, and divergently manifest these effects with improved tumor oxygenation. Next, we noted that the hyperoxia-induced reduction in tumor burden was not merely due to greater PMN accumulation within the tumor epithelium, since uterine PMNs in hyperoxia-housed PRPL mice instead assumed a more stromal distribution (Supplemental Figure 6, D-F). Thus, to identify the hyperoxia-regulated mechanisms of PMN-induced tumor cell death (i.e., sloughing) and proliferation, we evaluated PRPL mice with genetic lesions preventing generation of PMN products previously linked to cancer and basement membrane degrada- tion, namely PRPL-Elane ${ }^{-/-}$mice deficient in NE, PRPL-Mmp9 $9^{-1-}$ mice deficient in MMP-9, and PRPL-Cybb-/ mice deficient in the gp91 ${ }^{\text {phox }}$ subunit of the NADPH oxidase complex expressed by PMNs (NOX2) and used by PMNs to produce high levels of ROS following activation $(3,12,14)$. As with PRPL-Csf3r/ mice, these 3 additional mouse strains all showed the same tumor burdens as PRPL mice at the start of hyperoxia exposure on P18 (Supplemental Figure $6 \mathrm{G}$ ). Interpretively, we considered the amount of denuded endometrial surface to be a positive function of the level of tumor cell sloughing, since tumor cell sloughing generates these surfaces, but a negative function of tumor cell proliferation, since proliferation-induced reepithelialization would be expected to promote their disappearance.

Most straightforwardly, exposure of PRPL-Elane ${ }^{-1}$, PRPL$\mathrm{Cybb}^{-/}$, and PRPL-Mmp $9^{-/-}$mice to respiratory hyperoxia had no effect on their respective tumor burdens, thus indicating that NE, NOX2-derived ROS, and MMP-9 were all involved in the influence of hypoxia over net tumor growth (Figure 4, A-I). However, whereas the tumor burdens of normoxia-housed PRPL-Cybb-1and PRPL-Mmp $9^{-/-}$mice were similar to those of PRPL mice, the tumor burdens of PRPL-Elane ${ }^{-/}$mice were greatly reduced. This 

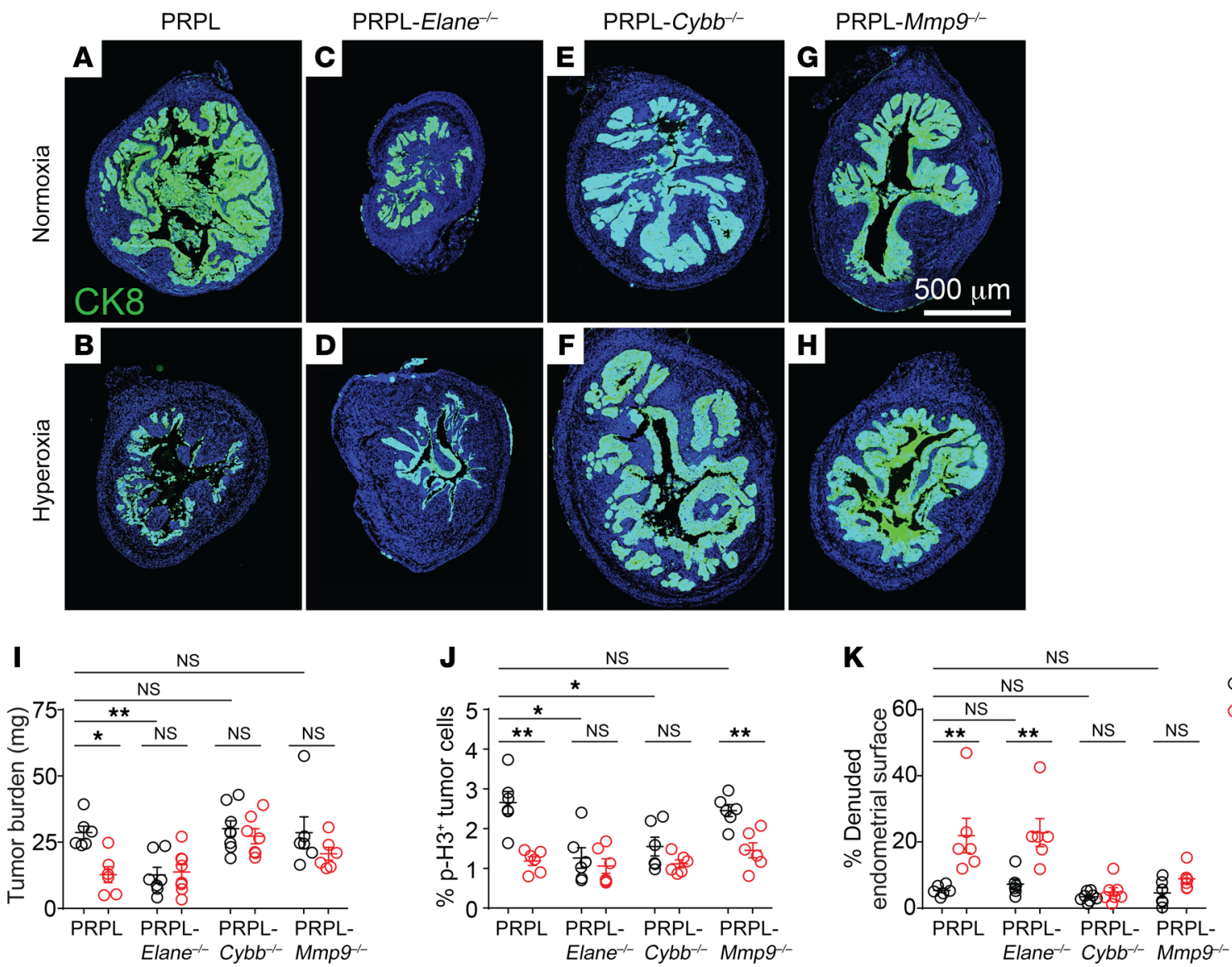

ONormoxia

OHyperoxia

$\mathbf{L}$

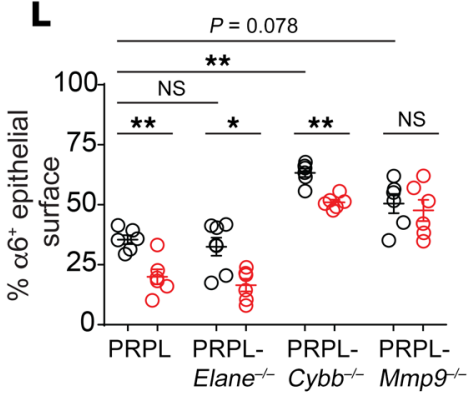

$\mathbf{N}$

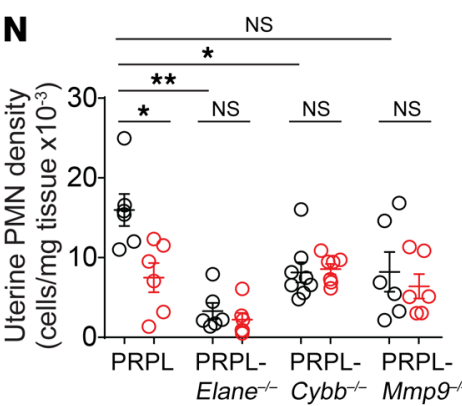

Figure 4. Role of NE, neutrophil-derived ROS, and MMP-9 in PMN control over tumor cell death and proliferation. (A-H) Representative CK8-stained

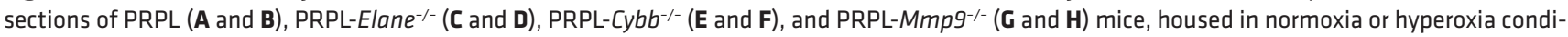
tions ( $n=6-8$ mice/group). These are the same sections for which $\alpha 6$ costaining is shown in Supplemental Figure 5. Quantification of (I) tumor burden, (J) tumor cell proliferation, (K) percentage of denuded endometrial surface, (L) percentage of tumor epithelial surface with basolateral $\alpha 6$ integrin staining, (M) percentage of sloughed tumor cells, and (N) uterine PMN tissue densities, as determined by flow cytometry. The data for PRPL mice are the same as in Figures 1-3. See Supplemental Figure 5 for representative images of $\alpha 6$ immunostaining. Graphs also show the mean $\pm S E M$. ${ }^{*} P<0.05$; ${ }^{* *} P<0.01$ by 2-tailed Mann-Whitney $U$ test. Differences between normoxia data were first assessed by the Kruskal-Wallis test $(P<0.01)$ and $P$ values were Bonferroni adjusted for multiple comparisons.

reduction could be attributed to a low level of tumor cell proliferation, which was evident under both normoxia and hyperoxia conditions and was moreover unaffected by hyperoxia housing (Figure 4J). Together, these results suggested that NE fostered tumor cell proliferation, similar to its previously documented effect in lung cancer (14), and that relief from tumor hypoxia decreased tumor cell proliferation in PRPL mice in part by attenuating this influence. Consistent with this possibility, hyperoxia-induced tumor cell sloughing appeared NE independent, since hyper- oxia housing, despite having no effect on tumor proliferation in PRPL-Elane ${ }^{--}$mice, increased their percentage of denuded surface and decreased their $\alpha 6$ basolateral staining (Figure 4, $\mathrm{K}$ and $\mathrm{L}$, and Supplemental Figure 5, E and F). On the other hand, hyperoxia housing did not increase the amount of sloughed tumor cells in PRPL-Elane ${ }^{-/}$mice (Figure 4M), as might have been expected, but we considered it unlikely that changes in sloughing would be detectable when tumor burden was so low and potentially only highly adherent cells remained attached to the endometrial stro- 
mal surface. A similar consideration likely explained why hyperoxia housing did not further decrease tumor burden in PRPL-Elane ${ }^{-/-}$ mice (Figure 4, C, D, and I).

We next noted that tumor cell proliferation in PRPL-Cybb ${ }^{-1-}$ mice was also low, like in PRPL-Elane ${ }^{-/}$mice, and was similarly unaffected by hyperoxia housing (Figure 4J). This suggested that NOX2-derived ROS also promoted PRPL tumor cell proliferation and that this influence waned when the tumor was rendered less hypoxic. However, since the tumor burdens of normoxia-housed PRPL-Cybb $b^{-/}$mice were similar to those of PRPL mice, their low tumor cell proliferation rate must have been canceled out by a concomitant reduction in tumor cell sloughing. Consistent with this possibility, $\alpha 6$ basolateral staining was dramatically increased in normoxia-housed PRPL-Cybb-/- (versus PRPL mice; Figure $4 \mathrm{~L}$ and Supplemental Figure 5, A and G). Moreover, hyperoxia housing only mildly affected this high level of staining and had no effect on percentage of denuded surface or amount of intraluminal debris (Figure 4, K-M, and Supplemental Figure 5, G and H). Similarly, PRPL-Mmp9-/- mice showed no change in percentage of denuded surface, amount of intraluminal debris, or $\alpha 6$ basolateral staining following hyperoxia housing, with their $\alpha 6$ basolateral staining under normoxia housing conditions marginally increased over that of PRPL mice $(P=0.078)$ (Figure $4, \mathrm{~K}-\mathrm{M}$, and Supplemental Figure 5, A, I, and J). Together, these observations suggested a dual requirement for NOX2-derived ROS and MMP-9 in tumor cell killing, particularly under conditions of improved tumor oxygenation. Tumor cell proliferation in PRPL- $\mathrm{Mmp9}^{-/-}$mice remained similar to that in PRPL mice and sensitive to respiratory hyperoxia, suggesting that it was not controlled by MMP-9 in a major way (Figure 4J). Of interest, NE deficiency reduced PMN tissue densities in PRPL tumors, while NOX2 and MMP-9 deficiencies abrogated the response of these densities to respiratory hyperoxia (Figure 4N). Most likely, these latter findings reflected the respective impact of the Elane, $C y b b$, and $M m p 9$ mutations on tumor burden and thus intrinsic levels of tumor hypoxia, combined with the aforementioned ability of PMNs to promote hypoxia and inflammation and thus their own recruitment in feed-forward fashion.

These mouse mutants also provided insight into how hyperoxia housing and tumor-associated PMNs controlled the advent of severe tumor hypoxia, as revealed through use of pimonidazole, a hypoxia-sensing reagent that becomes reactive when local tissue $\mathrm{O}_{2}$ levels fall below $1.3 \%$ (41). This was not a straightforward issue, since the percentage of pimonidazole ${ }^{+}$tumor area in PRPL-Csf3rmice was not reduced when the mice were housed in hyperoxia conditions (Supplemental Figure 7, A, J, and K), despite the tumor cells showing dramatically reduced nuclear HIF-1 $\alpha$ staining, as described above (Figure 1, D-F). These observations suggested a disconnect between the processes that were inducing the moderate levels of hypoxia (i.e., $\mathrm{O}_{2}$ below $\sim 6 \%$ ) sufficient to induce HIF-1 $\alpha$ activation (42) and the processes that were inducing severe hypoxia, as revealed by the pimonidazole reaction. As discussed further below, however, we noted that the percentages of pimonidazole ${ }^{+}$tumor cells across all mouse groups analyzed (Supplemental Figure 7) were elevated only when the data above suggested high tumor cell exposure to NE, and not in cases of high tumor cell proliferation, high ROS production by PMNs, or low levels of tumor cell killing.
Hypoxia alters the transcriptional signature of tumor-associated PMNs. To gain greater insight into how hypoxia altered the functionality of tumor-associated PMNs, we performed RNA sequencing (RNA-Seq) on PMNs sorted from the uteri of PRPL mice housed in hyperoxia and normoxia conditions. Of the 423 protein-coding genes that were differentially expressed (Figure 5A and Supplemental Table 1), some of the more interesting genes became clear when we visualized differential expression in terms of absolute changes in normalized read counts (Figure 5B). One gene upregulated in PMNs from hyperoxia uteri was $C y b b$, consistent with prior results with mouse PMN-MDSCs (26) and suggestive of greater ROS production. These cells also expressed higher levels of $M m p 2$ and Mmp14, which encode key activators of MMP-9 (43). Mmp9 itself was highly expressed by tumor-associated PMNs, as expected (5), but not differentially expressed (data not shown). Indeed, gelatin zymography performed on PRPL uterine extracts revealed similar total MMP-9 levels (active plus inactive forms) when the mice were housed in hyperoxia conditions, but a greater amount of the active form (Figure 5, C and F). Since hyperoxia conditions reduced PMN densities by approximately 60\% (Figure 1A), this increase likely underestimates the amount of active MMP-9 generated by PMNs on a per-cell basis. Irrespective of housing condition, uterine MMP-9 levels in PRPL mice were much higher than in PRPL-Csf3r-- mice (Figure 5C), indicating that PMNs were a major source of MMP-9 in PRPL tumors, in accord with our prior immunofluorescence analysis (5). Moreover, hyperoxia housing did not increase MMP-9 activation in the uteri of PRPL-Cs $3 \mathrm{rr}^{-/}$mice (Figure 5D). This observation indicated that the elevation in MMP-9 activity seen in the uteri of hyperoxia-housed PRPL mice involved the PMN-dependent component of its production and activation, and was consistent with the hyperoxia housing-induced increase in the cells' expression of Mmp2 and Mmp14.

Given the recent description of HLA class II-expressing PMNs in human cancer (9), we also noted the upregulated expression of genes related to peptide-MHC class II (MHCII) complex generation and costimulation, including $C d 74$, Ciita, $\mathrm{H2}-\mathrm{Ab1}$, $\mathrm{H2}-\mathrm{Aa}$, H2-Eb1, and Cd4O (Figure 5, A and B). With respect to downregulated genes, Siglecf stood out (Figure 5B) given the recent identification of SiglecF as a marker of tumor-promoting PMNs in lung cancer (8). Elane transcripts were undetectable under both normoxia and hyperoxia conditions, consistent with this gene's transcription during granulopoiesis (44); however, casein zymograms revealed decreased intrauterine $\mathrm{NE}$ activity following hyperoxia housing (Figure 5, E and G). These zymograms also revealed a total lack of NE activity in the uteri of PRPL-Csf3r-- mice, indicating that the source of this enzyme was exclusively PMNs. Of interest, the transcriptional changes in uterine PMNs isolated from hyperoxia- versus normoxia-housed PRPL mice did not bear an obvious relationship to the differences between N1 and N2 PMNs (45). Moreover, only a limited number of the differentially expressed genes we identified were present in a recent comparison between immunosuppressive PMN-MDSCs from cancer patients and their normal PMN counterparts (46), and these genes (e.g., Cpvl, S100a10, Ahnak, and genes encoding MHCII molecules) were all expressed at respectively higher levels in both PMN-MDSCs and hyperoxia-exposed PMNs, despite the apparently contrasting biological properties of these cell types. 
A
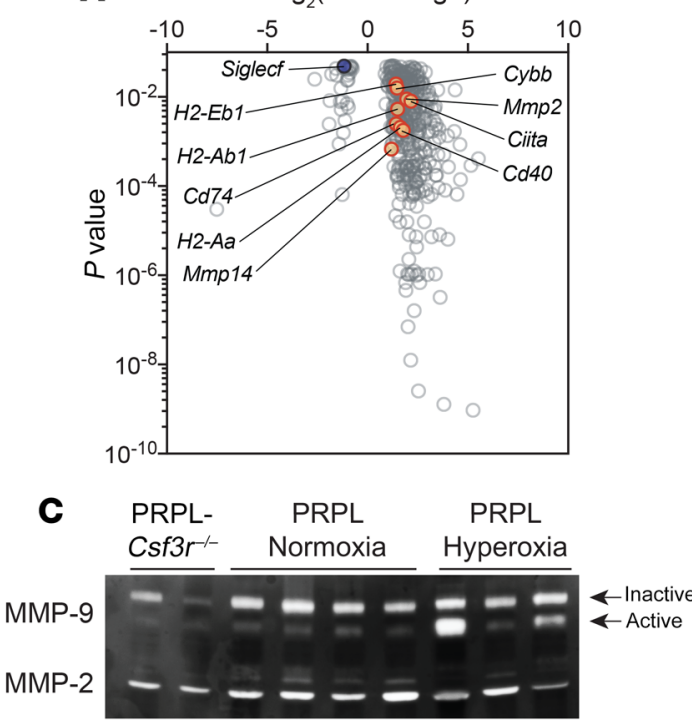

B $\quad \log _{10}($ abs change)
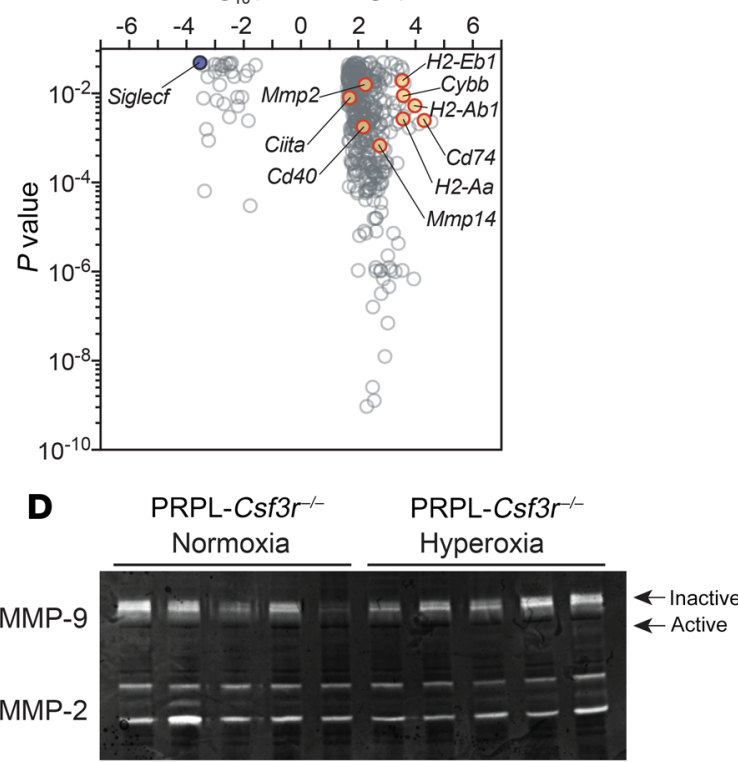

E

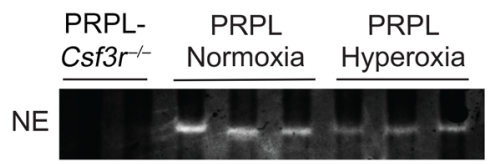

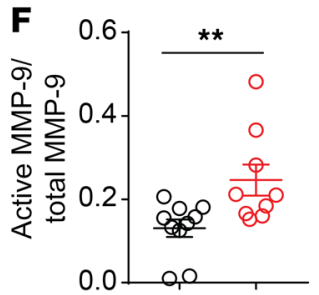

Figure 5. Relief of tumor hypoxia alters the transcriptional signature of tumor-associated PMNs and their production of active MMP-9 and NE. (A) Volcano plot showing the 423 differentially expressed protein-coding genes (FDR $<0.05)$ in PMNs isolated from PRPL mice housed under normoxia versus hyperoxia conditions ( $n=3$ per group). We excluded genes whose maximal average normalized reads in both of the 2 groups was less than 50 . (B) Volcano plot of this same gene set but with the $x$ axis showing the $\log _{10}$ of the absolute difference in mean normalized reads, to accentuate genes that are more differentially expressed in absolute terms. (C-E) Gelatin and casein zymograms performed on uterine extracts. Each lane represents a different mouse. Equal protein amounts were loaded per well. ( $F$ and $\mathbf{G})$ Quantification of MMP-9 and NE band intensities. Graphs also show the mean \pm SEM. ${ }^{* *} P<0.01$ by 2 -tailed Mann-Whitney $U$ test. In addition to the 2 gels shown ( $\mathbf{C}$ and $\mathbf{E}$ ), these data came from extracts run in 2 additional gelatin zymograms and 1 additional casein zymogram.

PMNs exist as malleable, hypoxia-regulated subpopulations in the tumor microenvironment. Capitalizing on these data, we performed flow cytometry on disaggregated uterine tissues to further determine how hypoxia altered PMN phenotypes within the tumor microenvironment. As a point of comparison, we also assessed PMNs recruited to non-tumor-bearing uteri. Recruitment was accomplished by injecting LPS 24 hours prior to sacrifice into the uterine lumen of 4-week-old phenotypically wild-type "PL" mice bearing floxed alleles of Pten but no Pgr-Cre transgene. Remarkably, LPS injection induced the sloughing of the uterine epithelium, and this was primarily due to the recruited PMNs since it was much less apparent in LPS-injected PL-Csf3r/- mice (Figure 6, A-C, and Supplemental Figure 8A). For both PRPL and LPSinjected PL mice, uterine but not blood PMNs divided into CD11b $\mathrm{b}^{\text {lo }}$ versus $\mathrm{CD} 11 \mathrm{~b}^{\text {hi }}$ subsets at an approximately $80 \% / 20 \%$ relative proportion that was unaffected by hyperoxia housing (Figure 6D and Supplemental Figure 8B). Both subsets, moreover, contained cells that were producing ROS as well as cells expressing SiglecF. Strikingly, hyperoxia housing altered the expression of these 2 markers, but this effect was evident only in PRPL mice and was much more apparent with the CD11b ${ }^{\text {lo }}$ subset (Figure 6, E-G, and Supplemental Figure 8, C-E). For this subset, hyperoxia housing reduced the proportion of $\mathrm{ROS}^{-}$Siglec $\mathrm{F}^{+}$cells, increased the proportion of $\mathrm{ROS}^{+}$ SiglecF $\mathrm{F}^{-}$cells, and reduced SiglecF expression levels on $\mathrm{ROS}^{+}$ SiglecF $F^{+}$double-positive cells. CD11b hi uterine PMNs, by contrast, showed only statistically insignificant changes (albeit with directionally parallel trends) and were much more $\mathrm{ROS}^{+}$SiglecF ${ }^{+}$double positive to begin with in PRPL mice (Supplemental Figure 8, C-E). Blood PMNs showed no ROS production and their expression of SiglecF, which was detectable on approximately $20 \%$ of the cells in both PRPL and LPS-injected PL mice, was unchanged by hyperoxia housing (Supplemental Figure 8, F and G).

These observations were consistent with the Cybb and Siglecf expression changes detected by RNA-Seq. We also found that hyperoxia housing increased the level of MHCII expression by tumor-associated PMNs, also consistent with the RNA-Seq analysis. In this case, however, it was the CD $11 b^{\text {hi }}$ and not the CD $11 b^{\text {lo }}$ subset that was affected, but similar to our results with ROS production and SiglecF expression, the shift was not apparent in blood PMNs nor in PMNs recruited to the uterus by LPS (Supplemental 

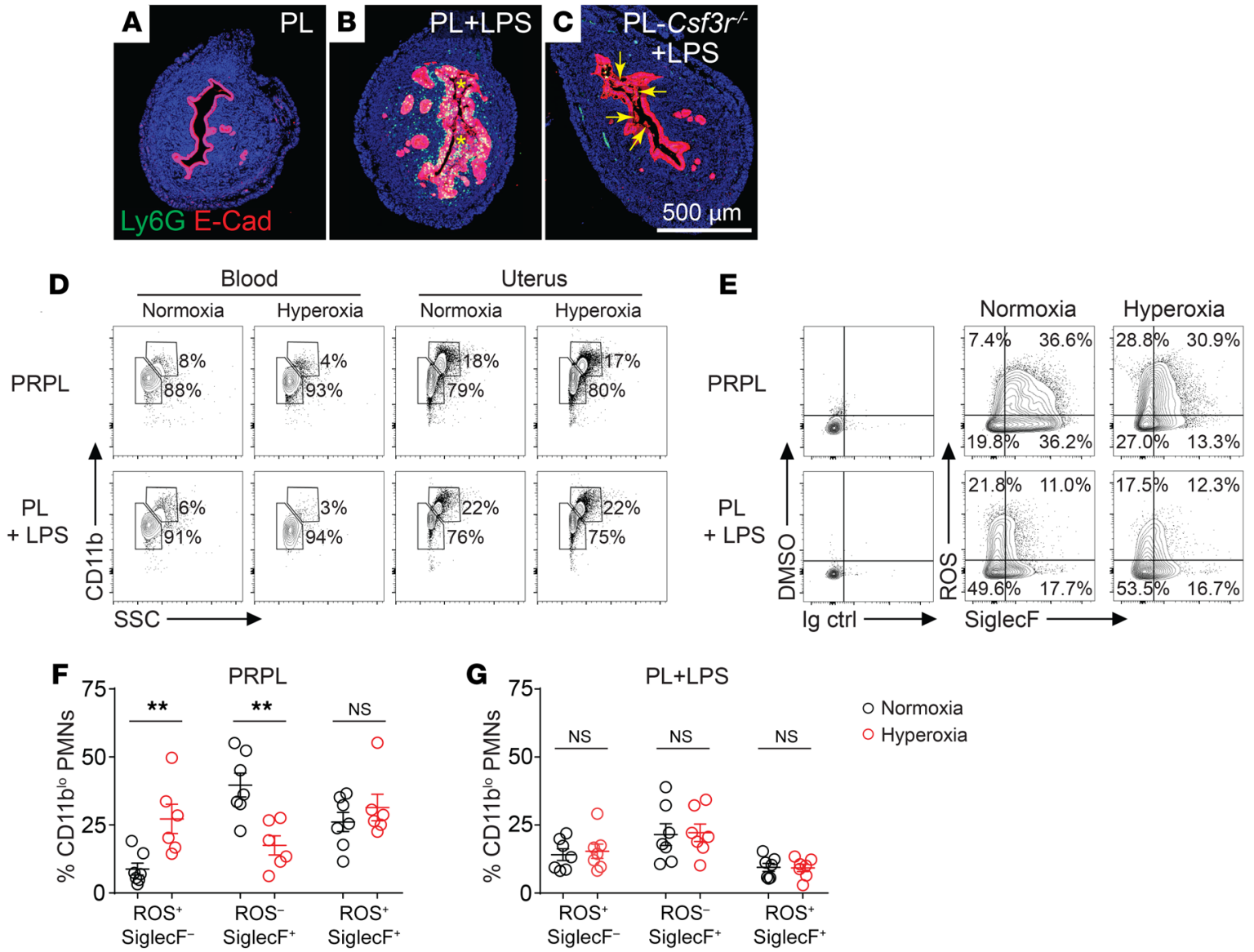

Figure 6. Relief of tumor hypoxia alters the phenotype of tumor-associated PMNs. PRPL mice were subjected to hyperoxic housing for 10 days prior to sacrifice on P28; PL and PL-Csf3r-1- mice received intrauterine injections of LPS 24 hours prior to sacrifice on P28. Some of the PL mice were also subjected to hyperoxic housing for this 24-hour period. (A-C) Representative E-Cad-stained uterine cross sections ( $n=5-8$ mice/group). Asterisks (B) and arrows (C) indicate intraluminal debris; see Supplemental Figure 8A for its quantification. (D) Representative flow cytometry plots of Ly6G hi cells from the blood and uteri ( $n=6-7$ mice/group, see Supplemental Figure 8B for quantification of uterine PMNs). (E) Representative flow cytometry plots showing ROS production and SiglecF expression by uterine CD11b ${ }^{10}$ PMNs ( $n=6-7$ mice/group). ROS production was determined through use of dihydrorhodamine 123 , a cell-permeant fluorescent ROS detection reagent. DMSO is the solvent for this reagent. (F and $\mathbf{G}$ ) Flow cytometric quantification of ROS production and SiglecF expression by CD11 $\mathrm{b}^{10} \mathrm{PMNs}$. Cells were gated on live/CD45 $/$ Ly6 $\mathrm{C}^{\mathrm{hi}}$ events, and then were subdivided according to CD11b expression levels (see Supplemental Figure 8, C-E, for analysis of the CD11 b ${ }^{\text {hi }}$ cells). Graphs also show the mean \pm SEM. ${ }^{* *} P<0.01$ by 2-tailed Mann-Whitney $U$ test.

Figure 9, A-D). Together, these results suggested that the phenotype of tumor-associated PMNs remains malleable and that hyperoxia housing altered this phenotype, at least in part by relieving tumor hypoxia and not by having some kind of de novo and potentially artifactual or systemic effect on PMNs. Consistent with this possibility, hyperoxia housing did not augment epithelial sloughing in LPS-injected PL mice (Supplemental Figure 8A). Importantly, we cannot formally rule out the possibility that hyperoxia housing had additional effects on PMNs prior to their extravasation into the tumor-bearing uterus; however, our observation that blood PMNs in PRPL mice had a uniformly Ly6 $\mathrm{G}^{\mathrm{hi}}$ surface phenotype following both normoxia and hyperoxia housing (Supplemental Figure 1, $\mathrm{F}-\mathrm{H}$ ) argues against hyperoxia exposure inducing emergency granulopoiesis and thus a shift toward an immature PMN phenotype. On the other hand, and consistent with an effect of the tumor per se on granulopoiesis, PMN frequencies in the blood are mildly elevated in the blood of 4-week-old PRPL compared with PL mice (5), while approximately $5 \%$ of blood PMNs in PRPL but not PL mice expressed MHCII (Supplemental Figure 9, C and D).
Of note, levels of SiglecF expression by PMNs within PRPL and LPS-injected PL uteri barely reached those of eosinophils in the same specimens (Supplemental Figure 9E), thus distinguishing SiglecF ${ }^{+}$PMNs in 4-week PRPL tumors from lung cancer-associated PMNs, whose SiglecF expression levels are similar to that of eosinophils (8). Provocatively, however, SiglecF expression by uterine PMNs was much more pronounced when the cells were isolated from 12-week-old PRPL mice, thus suggesting an effect of tumor stage on PMN phenotypes (Supplemental Figure 9, F and G).

PMNs, employing both NOX2 and mitochondria, constitute the vast majority of ROS-producing cells in PRPL tumors, but hypoxia only regulates ROS production by NOX2. Given that the $2 \mathrm{PMN}$ subsets in PRPL tumors showed different behaviors with respect to ROS production, we next determined the extent to which these 2 subsets produced ROS via NOX2 and thus might contribute toward the NOX2-dependent tumor cell killing we documented using PRPL-Cybb $b^{-/}$mice. As expected from the above flow analysis, hyperoxia housing increased ROS production by CD11b ${ }^{\text {lo }}$ PMNs (now viewing the cells in aggregate independently of 

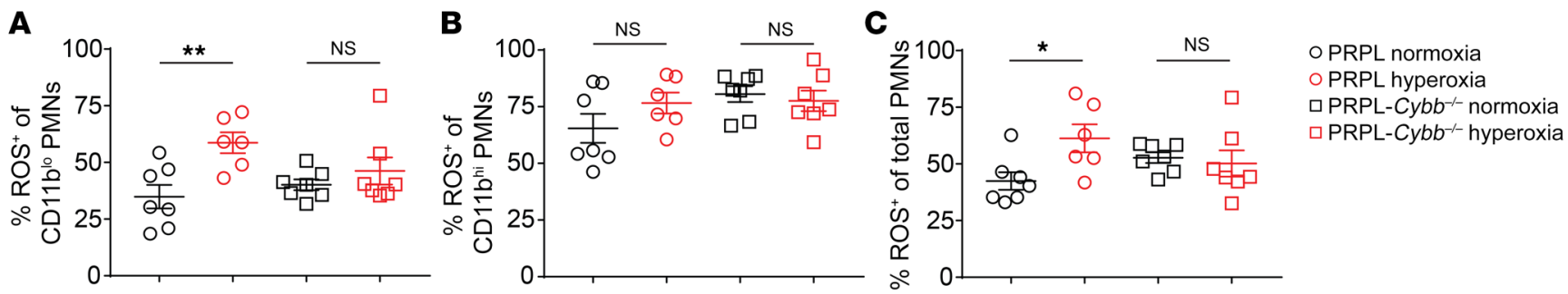

D
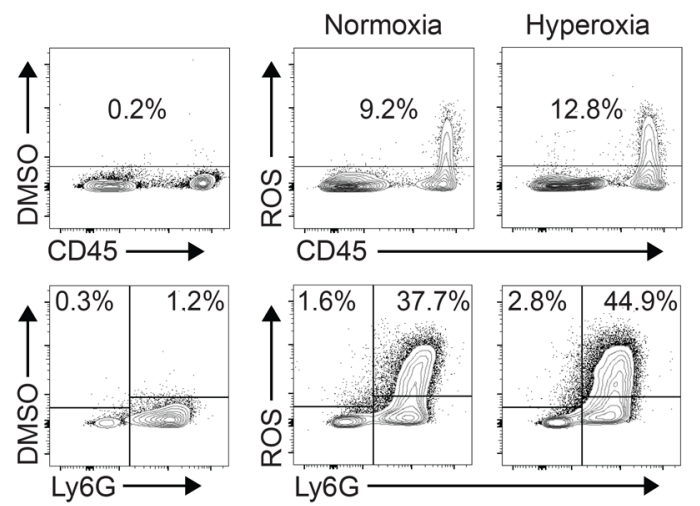

E

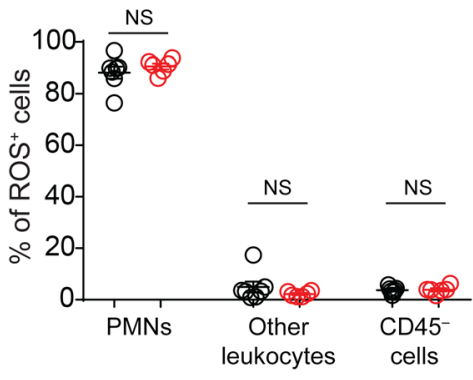

O Normoxia

O Hyperoxia

Figure 7. Relief of tumor hypoxia increases NOX2-dependent ROS production by tumor-associated PMNs. (A-C) Flow cytometric assessment of ROS production (using dihydrorhodamine 123 ) by the indicated PMN subsets. (D) Representative flow cytometry plots ( $n=6-7$ mice/group; bottom plots are gated on $\mathrm{CD} 45^{+}$cells) and (E) quantification of ROS production by all cell types within $\mathrm{PRPL}$ uteri. Graphs also show the mean $\pm \mathrm{SEM}$. ${ }^{*} P<0.05$; ${ }^{*} P<0.01$ by 2-tailed Mann-Whitney $U$ test.

SiglecF expression levels). This increase was dependent on NOX2, as it was not apparent in CD $11 b^{\text {lo }}$ PMNs isolated from the uteri of hyperoxia-housed PRPL-Cybb $b^{-/}$mice (Figure 7A). Unexpectedly, however, ROS production by CD11b ${ }^{\text {lo }}$ PMNs from PRPL-Cybb $b^{-/}$ mice was still quite substantial ( $40 \%$ positive cells) and similar to that of $\mathrm{CD} 11 \mathrm{~b}^{\text {lo }} \mathrm{PMN}$ from PRPL mice housed under normoxia conditions (Figure 7A). A similar pattern was evident with CD11b $\mathrm{b}^{\text {hi }}$ PMNs (which in general produced more ROS than their CD11b $b^{\text {lo }}$ counterparts), although their hyperoxia-induced increase in ROS production, following their isolation from PRPL mice, was more modest in terms of fold induction (and did not reach statistical significance; Figure 7B). NOX2-dependent ROS production following hyperoxia housing was also evident when all PMNs were considered together (Figure 7C). Importantly, ROS production via mitochondrial respiration is known to be elevated in PMNs isolated from human patients with $C y b b$ deficiencies (47), thus precluding rigorous interpretation of these data with respect to the relative contributions of NOX2 versus mitochondrial respiration to ROS production by tumor-associated PMNs in PRPL tumors; however, they are consistent with the possibility that mitochondrial respiration and not NOX2 is the dominant source of PMN-derived ROS in the PRPL tumor microenvironment under normoxia housing conditions. Moreover, we found that PMNs constitute the vast majority ( $90 \%)$ of ROS-producing cells in the PRPL tumor microenvironment under both normoxia and hyperoxia housing conditions, with the amount of ROS produced by PMNs on a per-cell basis also greatly exceeding all other cell types (Figure 7, D and E). Together, these data suggest that mitochondrial respiration by PMNs is the major source of ROS in the PRPL tumor microenvironment, particularly when the tumor is allowed to remain hypoxic, and that stimulation of NOX2 activity in PMNs upon relief of tumor hypoxia generates the ROS that promotes tumor cell sloughing.

\section{Discussion}

A key question that arises when considering the role of PMNs in tumorigenesis is why their effects appear so context dependent. Here, using an autochthonous mouse model of endometrial cancer and the administration of respiratory hyperoxia to improve tumor oxygenation, we provide in vivo evidence that hypoxia exerts a powerful but complex influence over tumor-associated PMN behavior and phenotypes. In the most general terms, we found that hypoxia promoted PMN recruitment to the tumor-bearing uterus but then prevented the cells from reaching their maximal tumor-combatting potential. These divergent effects, however, were unequally balanced, as the reduction in PMN recruitment to the tumor-bearing uterus achieved via the administration of respiratory hyperoxia was more than compensated for by the augmented ability of the recruited cells to oppose tumor growth. Thus, relieving tumor hypoxia greatly improved net PMN-dependent tumor control and caused a massive reduction in tumor burden. Importantly, improved tumor control in this tumor model was completely $\mathrm{T}$ cell independent and instead reflected alterations in the direct interactions between PMNs and tumor cells. Given the variable presence of hypoxia in different tumor settings, these data thus suggest one way that the context-dependent effects of PMNs in cancer might be understood. Importantly, these hypoxiaregulated interactions may have less relevance to nonepithelial cancers given the basement membrane-dependent killing mechanism we describe here. Moreover, with the increasingly recognized possibility that hypoxia can augment the ability of PMN/PMN- 
MDSCs to suppress $\mathrm{T}$ cells $(26,33)$, the ultimate link between hypoxia-regulated PMNs and clinical outcome is likely to be explained through a combination of $\mathrm{T}$ cell-dependent and $\mathrm{T}$ cellindependent pathways. Indeed, our ability to gather evidence in support of direct hypoxia-regulated PMN-tumor cell interactions may have been facilitated by the fact that PRPL tumors are not controlled by T cells at their early stage of development (5).

Our results suggested that hypoxia promotes PMN recruitment to 4-week PRPL lesions, at least in part by augmenting CXCR2 ligand production by the tumor cells, an effect apparent in vivo and in the absence of PMNs themselves. These observations are consistent with our prior data correlating the onset and spatial distribution of hypoxia in PRPL tumors with the onset of PMN recruitment and their localized aggregation within the tumor, respectively, as well as with our prior observation that hypoxia induces the expression of IL-8, a human PMN chemoattractant, in cultured human endometrial carcinoma cells (5). Interestingly, we also found that hypoxia induces STAT3 phosphorylation in PRPL tumor cells in vivo, and that STAT3 is necessary for CXCL5 expression and PMN recruitment, but the exact pathway that connects hypoxia to CXCL5 expression remains unclear. Similarly unclear is whether hypoxia is the sole STAT3 activator in PRPL lesions. As this seems unlikely given the many pathways that feed into STAT3, it is reasonable to assume that these other pathways also contribute to PMN recruitment to PRPL uteri.

Our data suggested that relief of tumor hypoxia inhibited PRPL tumor growth at least in part by limiting the ability of NE to induce tumor cell proliferation. In previous work on lung cancer, this proproliferative effect was ascribed to NE uptake by tumor cells and consequent modulation of mitogenic signaling pathways (14), but whether a similar mechanism applies here remains to be determined. Since Elane transcripts were undetectable in tumor-associated PMNs, however, the limiting effect of hyperoxia housing must have had posttranscriptional causes. Consistent with the results of our casein zymograms, one possibility is that the hyperoxiainduced reduction in uterine PMN densities meant that there were fewer cells available to release NE; a second is increased intrauterine expression of an $\mathrm{NE}$ inhibitor. It is also possible that the hyperoxia-induced redistribution of PMNs away from the tumor epithelium reduced tumor cell exposure to NE. The amelioration of tumor hypoxia might also have limited NE release from PMNs, consistent with the observed reduction in NE release from HIF-1 $\alpha-$ deficient murine PMNs (24) and the ability of hypoxia, conversely, to promote NE release from human PMNs (22). Interestingly, our observations using pimonidazole as a hypoxia detection reagent also implicates NE in generating severe tumor hypoxia. We speculate that this effect might be the consequence of the ability of $\mathrm{NE}$ to cause vascular injury (48), which might generate focal areas of ischemia superimposed over the background levels of hypoxia that arise from the metabolic demands of the dividing tumor cells. These observations also raise the question of how frequently other kinds of tumors might show discrepant measurements of HIF- $1 \alpha$ activation versus pimonidazole reactivity.

Relief of tumor hypoxia also inhibited tumor growth by promoting PMN-induced tumor cell death. This component of the response was associated with increased tumor cell sloughing and appeared to be mediated by the combined activities of MMP-9 and NOX2-derived ROS. Moreover, our gelatin zymograms indicated that PMNs were the primary source of MMP-9 in PRPL tumors as well as the primary cell type responsible for increased MMP-9 activation under hyperoxia housing conditions, while our RNA-Seq analysis linked increased MMP-9 activation to increased PMN expression of MMP-2 and MMP-14. Similarly, our flow cytometric analysis revealed that PMNs were by far the major ROS producers in PRPL tumors and that hyperoxia housing increased ROS production by PMNs on a per-cell basis and in a NOX2dependent fashion. This increase paralleled the cells' increased expression of $C y b b$ mRNA. These latter observations are consistent with the previously described ability of hypoxia, conversely, to inhibit $C y b b$ expression and respiratory burst activity in isolated mouse PMN-MDSCs (26) and to inhibit respiratory burst activity in human PMNs (25). Together, these data thus suggest that hyperoxia housing increased the net exposure of the tumor epithelium to active MMP-9 and NOX2-derived ROS, both generated primarily by PMNs, even while it reduced uterine PMN densities and redistributed the cells away from the tumor epithelium. Despite evidence that PMN-derived ROS can directly kill tumor cells in vitro $(17,49)$, our previous finding that the cellular debris within the uterine lumen of PRPL mice contains many live tumor cells makes us suspect that the primary mechanism of tumor cell death is basement membrane degradation leading to tumor cell detachment from the endometrial stroma. Indeed, basement membrane degradation is another in vitro effect of $\operatorname{ROS}(50,51)$, while MMP-9 can also degrade basement membranes in vitro and promotes epithelial cell detachment from basement membranes in the context of bullous pemphigoid disease in vivo $(12,13,15)$. Moreover, basement membrane degradation is consistent with our ability to discern PMN-induced tumor cell sloughing via NOX2-derived ROS over a high background of PMN-derived mitochondrial ROS, as $\mathrm{H}_{2} \mathrm{O}_{2}$ generated by NOX2 but not by mitochondria has the capacity to be converted by granule-associated myeloperoxidase into hypochlorous acid, which is particularly degradative for ECM molecules (52). Basement membrane degradation is also consistent with the loss of $\alpha 6 \beta 4$ polarization from the tumor cell basolateral surface, particularly given our prior observation that tumor cells from PRPL and PRPL-Cs $3 \mathrm{rr}^{-/}$mice express the same levels of total surface $\alpha 6 \beta 4$ (5). NOX2-derived ROS also appeared to promote tumor cell proliferation, with a similar degree of reduction in tumor cell proliferation to that seen in PRPL-Cybb-/- and PRPLElane $^{-/}$mice, suggesting an interaction between such ROS and NE. Indeed, previous work suggests that respiratory burst activity is required for NE release from azurophilic granules, at least in the context of NETosis (53).

Provocatively, improved tumor oxygenation also increased uterine PMN transcript levels of genes involved in peptide-MHCII complex generation and costimulation, an effect confined to the CD11b ${ }^{\text {hi }}$ PMN subset. Given that these cells expressed high levels of ROS and so might be highly activated, it is unclear whether they themselves were transcriptionally altered by hyperoxia housing, or whether hyperoxia housing differentially promoted their survival within the uterus following their recruitment from the blood, where $\mathrm{MHCII}^{+}$PMNs were enriched in PRPL mice compared with non-tumor-bearing controls. Either way, the existence of these $\mathrm{MHCII}^{+}$cells relate our findings to an emerging popula- 
tion of $\mathrm{MHCII}^{+} \mathrm{PMNs}$ with antigen-presenting capacity previously identified in mice in the context of inflammation (54) and in human lung cancer specimens (9). Interestingly, hypoxic culture conditions were shown to prevent the generation of such "TAN hybrids" from human precursors in vitro, an observation that was used to explain why these cells were less prevalent in larger (i.e., more hypoxic) tumors in vivo (9). Similarly, the expression of SiglecF by PRPL tumor-associated PMNs relate our findings to the SiglecF $F^{\text {hi }}$ PMNs recently described to populate murine models of lung cancer and that are thought to have tumor-promoting properties (8). Intriguingly, PMNs in the tumors of 4-week-old PRPL mice expressed much lower levels of SiglecF, on average, than the PMNs in these lung tumors and only strongly upregulated SiglecF at more advanced stages of tumor development. Moreover, hyperoxia housing reduced SiglecF expression in 4-week PRPL tumors as it increased the tumor-killing potential of the cells. These observations further suggest the potential of using SiglecF expression as a marker of tumor-promoting PMNs and raise questions about its potential causative role in fostering such protumor functions.

Importantly, the effects of hypoxia on tumor-associated PMN behavior and phenotypes likely reflect the complex outcome of both PMN-intrinsic and -extrinsic pathways. For example, PMN accumulation in PRPL tumors was at least in part an indirect effect of tumor hypoxia, as it appeared mediated by STAT3dependent production of CXCL5 (and possibly CXCL1 and CXCL2) by tumor cells, which in turn induced PMN recruitment. However, it has also previously been shown that hypoxia can directly extend PMN lifespan through cell-intrinsic, HIF- $1 \alpha-$ and HIF-2 $\alpha$-dependent pathways $(21,23)$. Similarly, altered PMN effector function under hypoxic conditions might reflect in part the direct effects of hypoxia on PMN degranulation and respiratory burst activity, as described above, but also its indirect effects as mediated through other cell types. For example, extracellular adenosine, which is generated in the hypoxic tumor microenvironment (55), can inhibit PMN respiratory burst activity (56). Further work will be necessary to determine the identity and relative importance of these intrinsic and extrinsic pathways over the control of tumor-associated PMN phenotypes.

Although the experiments performed here were motivated by our interest in how hypoxia controls tumor-associated PMNs, they also bear upon the question of how hypoxia influences solid tumor development more generally. Indeed, while tumor hypoxia is well known to have many effects on tumor cells and the tumor microenvironment $(30,57)$, it has been difficult to discern the relative importance of those effects since it has been difficult to control tumor oxygenation as the sole experimental variable. Thus, it is striking that altered PMN-tumor cell interactions are by far the dominant means through which relief of tumor hypoxia, achieved via the administration of respiratory hyperoxia, affects PRPL tumor growth. This dominance might reflect the high density of PMNs in PRPL lesions (5), or it might reflect, in principle, a lack of effect of hypoxia on the vascularity or the tumor cell-intrinsic growth characteristics of PRPL tumors. Since the lesions of 4-week-old PRPL mice are still at a relatively early stage of development, the net tumor growth-opposing effects of PMNs we document here may also reflect the possibility that PMNs become more overtly "protumor" only with advancing stages of tumor formation, a pos- sibility suggested by recent work on other tumor models $(4,9,11)$ and consistent with the cells' tumor stage-dependent expression level of SiglecF. Thus, with special consideration of tumor stage, these results suggest that greater attention to direct PMN-tumor cell interactions is warranted when considering the role of hypoxia in other kinds of cancers. Moreover, they inform the interpretation of recent studies suggesting that therapeutic relief of tumor hypoxia augments antitumor lymphocyte responses $(32,33)$, as these studies did not address the potential contribution of PMNs as direct antitumor effectors. Indeed, given our data here and our previous results (5) showing that PMNs can directly combat PRPL tumor formation independently of all lymphocytes and that this activity can be increased with improved oxygenation, it will be of interest to determine the extent to which this information might help with the development of hypoxia reduction as a therapeutic tool in other cancer settings.

\section{Methods}

Animals. PRPL (PgrCre/+ Pten $\left.^{f / f t}\right)$ mice on a C57BL/6 background were generated as previously described (5) from $\mathrm{Pgr}^{\mathrm{Cre}}$ mice (58) (the gift of Francesco DeMayo, Baylor College of Medicine, Houston, Texas, USA) and Pten $^{f / f l}$ mice (The Jackson Laboratory, stock 006440, referred to here as "PL" mice). PRPL mice were intercrossed with additional C57BL/6-background strains, namely Csf3r $r^{-/}$, Stat $3^{f / f l}$, Elane $^{-/-}$, Cybb $b^{-/-}$ (The Jackson Laboratory, stocks 017838, 016923, 006112 and 002365, respectively), and $\mathrm{Mmp9}^{-/-}$mice (59) (the gift of Zena Werb, UCSF). The mice were maintained in specific pathogen-free animal barrier facilities at UCSF (all experiments aside from those on PRPL-Stat $3^{d / d}$ mice) and the NYU School of Medicine (PRPL-Stat $3^{d / d}$ mice).

Treatments. For hyperoxia studies, PRPL mice were housed in a Small A-Chamber environmental chamber with ProOx P110 oxygen controller (BioSpherix) set at $60 \%$ oxygen but otherwise the ambient humidity and temperature of our mouse holding room. For T cell depletion, mice were injected i.p. with anti-CD4 antibodies (300 $\mu \mathrm{g}$; BioXCell, clone GK1.5) and anti-CD8 antibodies (200 $\mu \mathrm{g}$; BioXCell, clone 2.43) on P18, P21, P24, and P27. For LPS stimulation, PL female mice were injected transcervically 24 hours before sacrifice on P28 with $1.25 \mu \mathrm{g} / \mathrm{g}$ LPS from Salmonella enterica (serotype typhimurium, Sigma-Aldrich) dissolved in $10 \mu \mathrm{L}$ PBS. Transcervical injection was performed as previously described (60). For the in situ detection of severe tumor hypoxia, mice were injected i.p. with $0.75 \mathrm{mg}$ pimonidazole hydrochloride (Hypoxyprobe) 1.5 hours prior to sacrifice.

Flow cytometry. Dissected whole uteri were weighed and digested as previously described (60). Briefly, minced uteri were incubated in Hank's Balanced Salt Solution containing 0.28 Wünsch units/mL Liberase Research Grade (Roche) and $30 \mu \mathrm{g} / \mathrm{mL}$ DNAase I (Roche) for 30 minutes at $37^{\circ} \mathrm{C}$ with intermittent trituration. Tissues were then washed, resuspended in PBS $/ 1 \% \mathrm{FBS} / 5 \mathrm{mM}$ EDTA, incubated at $37^{\circ} \mathrm{C}$ for an additional 15 minutes, and then filtered through $40-\mu \mathrm{m}$ nylon mesh. We RBC-lysed blood samples using Gey's buffer.

The following flow cytometry antibodies were from BioLegend: CD8 $\alpha$ (clone 53-6.7); CD45 (clone 30F-11); CD11c (clone N418); Ly6G (clone 1A8); Ly6C (clone HK1.4); MHCII (I-A/I-E) (clone M5/114.15.2); F4/80 (clone BM8); EpCAM (CD326) (clone G8.8); ICAM2 (CD102) [clone 3C4 (MIC2/4)]; NK1.1 (clone PK136); Thy1.1 (clone 53-2.1); and rat IgG2b, $\kappa$ isotype control (clone RTK4530) (for MHCII staining). Antibodies against CD11b (M1/70), SiglecF (clone E50-2440), 
TCR $\gamma \delta$ (clone GL3), TCR $\beta$ (clone H57-597), and rat IgG2a, $\kappa$ isotype control (clone R35-95) (for SiglecF staining) were from BD Pharmingen. Antibodies against CD4 (clone RM4-5) were from Tonbo Biosciences. The cells were first incubated with rat anti-mouse CD16/CD32 (BioXCell, clone $2.4 \mathrm{G} 2 ; 5 \mu \mathrm{g} / \mathrm{mL}$ for $1 \times 10^{6}$ cells) to block nonspecific antibody binding. Live/dead discrimination employed $5 \mu \mathrm{g} / \mathrm{mL}$ 7-aminoactinomycin D (BD Pharmingen) or Fixable Viability Dye eFluor 506 (Invitrogen). Samples were analyzed using an 18-color 4-laser BD LSRFortessa cytometer or sorted on an 18-color 4-laser BD FACSAria II. CountBright beads (Invitrogen) were added to each sample to allow for leukocyte subset density calculations (cells/mg tissue). For ROS production, digested cell preparations, prior to antibody staining, were cultured at $5.0 \times 10^{5}$ cells $/ \mathrm{mL}$ in RPMI $/ 10 \% \mathrm{FBS}$ for 30 minutes at $37^{\circ} \mathrm{C}$ in a $5 \% \mathrm{CO}_{2}$ incubator in the presence of $12.5 \mathrm{ng} / \mathrm{mL}$ dihydrorhodamine 123 (Life Technologies) or vehicle control (0.025\% DMSO). Flow cytometric analysis employed FlowJo (Tree Star).

Immunofluorescence staining. Immunofluorescence staining of paraformaldehyde-fixed, paraffin-embedded tissue was performed as previously described (61). Depending on the primary antibody, tissue sections were subjected to antigen retrieval either by incubation in $1 \mathrm{mg} / \mathrm{mL}$ trypsin in $\mathrm{H}_{2} \mathrm{O}$ for 11 minutes at $37^{\circ} \mathrm{C}$, or by boiling in 0.01 $\mathrm{M}$ citric acid pH 6.0 (citrate) or 0.010 M Tris-EDTA pH 9.0 (TE) for 28 minutes in a pressure cooker. Slides were then blocked in $\mathrm{PBS} / 3 \%$ bovine serum albumin (BSA) (Sigma-Aldrich) $/ 3 \%$ donkey serum (Millipore)/0.4\% Triton X-100 (Sigma-Aldrich) for 1 hour at room temperature, and then incubated overnight at $4^{\circ} \mathrm{C}$ with primary antibodies diluted in PBS/1\% BSA/0.4\% Triton X-100. Antibodies, antibody dilutions, and antigen retrieval methods were as follows: CXCL5 (1:100, trypsin; Lifespan Biosciences, rabbit polyclonal, catalog LS-C212192); Ly6G (1:100, citrate or TE; BD Pharmingen, rat monoclonal, clone 1A8); integrin $\alpha 6$ (1:1000, TE; Abcam, rabbit monoclonal, clone EPR18124); histone H3 phospho-Ser10 (1:100, citrate; Cell Signaling Technology, rabbit polyclonal, catalog 9701); STAT3 phospho-Tyr705 (1:300, citrate; Cell Signaling Technology, rabbit polyclonal, cata$\log 9131)$; HIF-1 $\alpha$ (1:1000, TE; Novus Biologicals, rabbit polyclonal, catalog NB100-479B); NF-кB p65 phospho-Ser276 (1:3000, citrate; Abcam, rabbit polyclonal, catalog ab106129); CK8 (1:100, trypsin, citrate, or TE; University of Iowa, Developmental Studies Hybridoma Bank, rat monoclonal, clone TROMA-I); E-cadherin (1:1000, citrate or TE; Cell Signaling Technology, rabbit monoclonal, clone 24E10); CC-3 (1:100, citrate; Cell Signaling Technology, rabbit polyclonal, catalog 9661); pimonidazole (1:100, citrate; Hypoxyprobe, FITC-conjugated mouse monoclonal, clone 4.3.11.3); CD45 (1:100, citrate; BioLegend, rat monoclonal, clone I3/2.3); and TROP-2 (1:50, citrate; R\&D Systems, goat polyclonal, catalog AF1122).

After incubation with primary antibodies, the sections were incubated for 30 minutes at room temperature in the following secondary antibodies from Jackson ImmunoResearch: donkey anti-rat IgG-Alexa Fluor 488 (CK8 and Ly6G) or -Alexa Fluor 594 (integrin $\alpha 6$ ); donkey anti-goat IgG-Alexa Fluor 647 (TROP-2); donkey anti-rabbit IgGhorseradish peroxidase (HRP) (E-cadherin, p-H3, CXCL5, p-STAT3, HIF1 $\alpha$, and NF- $\mathrm{B}$ p65); or donkey anti-rat IgG-HRP (CD45). Secondary antibodies were diluted 1:200 either in PBS/1\% BSA/0.4\% Triton $\mathrm{X}-100$ or, for HRP-conjugated antibodies, in TNB blocking buffer (PerkinElmer). HRP-labeled slides were subjected to tyramide signal amplification in PBS $/ 1.8 \mu \mathrm{g} / \mathrm{mL}$ biotin-tyramide/0.0015\% $\mathrm{H}_{2} \mathrm{O}_{2}$ for 5 minutes at room temperature, and then incubated 30 minutes at room temperature with $5 \mu \mathrm{g} / \mathrm{mL}$ streptavidin-Alexa Fluor 594 or -Alexa Fluor 488 (Life Technologies) diluted in 1\% BSA. All slides were mounted using Fluoromount-G containing 4',6'-diamidino-2-phenylindole (DAPI) (Electron Microscopy Sciences).

Image acquisition, manipulation, and analysis. All immunofluorescence images were captured using an Axio Imager M2 and Zen software (Zeiss). Panoramic views were generated by tiling images taken with the $\times 10$ objective. For each primary antibody not revealing a structural feature of the tissue (i.e., antibodies against CXCL5, p-STAT3, HIF-1, NF-кB p65, integrin $\alpha 6$, Ly6G, pimonidazole, CC-3), images were respectively captured on the relevant fluorescence channel with the same exposure time and then subjected to the identical set of manipulations in Adobe Photoshop. Specifically, we first used the curves command to remove background fluorescence, and then the brightness/contrast command to achieve optimal visual discrimination across the set of images. For CK8, E-cadherin, TROP-2, CD45, and DAPI staining, brightness and contrast were optimized individually for each image. p-H3 staining was binary and thus unaffected by modest image manipulations. In order to improve the publication quality of panoramic images, we applied the maximum filter of ImageJ (NIH, https://imagej.nih.gov/ij/) for some stains and blurred the DAPI counterstain.

Our tumor burden calculations employed E-cadherin staining and a histomorphometric determination of intact cross-sectional tumor area. For this latter determination, as well as the determination of the amount of sloughed tumor cells, we first used the threshold command of Image J to highlight and then calculate the total area of all E-cadherin ${ }^{+}$staining (i.e., intact plus sloughed tumor area, determined for $3-5$ cross sections per mouse). We then repeated this procedure after first deleting regions of sloughed tumor, which were easily identified by eye because they were located within the lumen and had a macerated appearance, to determine the intact tumor area. For each cross section, sloughed area was then calculated as (total area) - (intact area), and percentage of sloughed area was calculated as (sloughed area)/(total area), then averaged over all cross sections analyzed for a given mouse. The percentage intact tumor area per total area of the cross section was also calculated, and this value's average over all cross sections analyzed for a mouse was then multiplied with the mouse's uterine weight to determine its tumor burden.

We used E-cadherin-stained sections and standard grid-based morphometry (62) to determine the amount of denuded endometrial surface. Specifically, percentage denuded surface was calculated as the number of times the grid intersected with a direct interface between the endometrial stroma and the uterine lumen (i.e., an interface with no covering epithelium), divided by the number of times the grid intersected with any kind of interface between uterine tissue and lumen (i.e., with or without a covering epithelium). Using a CK8 costain to identify the entire tumor epithelium, we similarly determined the percentage of basolateral tumor surface positive for $\alpha 6$ integrin staining. For both calculations, at least 3 entire cross sections were analyzed per mouse and then averaged.

For the CXCL5 quantification, we first used the CK8 costain and the Photoshop magic wand tool to identify all areas occupied by the tumor epithelium, then ImageJ to apply a uniform threshold across all images in order to binarize the CXCL5 staining. The percentage of CXCL5 ${ }^{+}$ tumor area was then calculated as the number of pixels positive for CXCL5 divided by the total number of pixels overlying tumor cells. An 
identical method was used to quantify pimonidazole staining, except that E-cadherin was used as the costain. The percentage of tumor cell nuclei staining positive for HIF-1 $\alpha$, p-STAT3, or NF- $\kappa$ B p 65 was similarly calculated; however, the CK8 and DAPI counterstains were together used to create a mask over all tumor cell nuclei. Determining the percentage of CC- $3^{+}$tumor cells again took a similar approach, since the extensive overlap between the CC-3 and DAPI staining allowed us to use the percentage of CC- $3^{+}$tumor cell nuclear area as a measure of the percentage of positive tumor cells. This analysis employed TROP-2/ DAPI staining to create the tumor cell nuclear mask and the CD45 costain to exclude leukocytes. Percentages of PMNs within the tumor epithelium were quantified histologically by binarizing Ly6G-stained images and then calculating the percentage of positive pixels within or without an epithelial mask defined by the E-cadherin costain. For all these analyses, at least 3 entire cross sections were analyzed per mouse and then averaged. For the determination of percentage of $\mathrm{p}-\mathrm{H}^{+}$ tumor cells, which were rare, we hand-counted $\mathrm{p}-\mathrm{H} 3^{+}$tumor cells and total $\mathrm{DAPI}^{+}$tumor cell nuclei over $3-5$ images $(\times 10$ magnification) per mouse, identifying tumor cell areas by CK8 costaining. At least 1500 total tumor cells were scored per mouse.

RNA-Seq. PMNs (CD45 ${ }^{+} \mathrm{Ly} 6 \mathrm{G}^{\text {hi }} \mathrm{CD} 11 \mathrm{~b}^{+}$cells) from disaggregated uteri (3 independent samples per group) were sorted on an 18-color 4-laser BD FACSAria II. RNA was then isolated using an RNeasy Micro Kit (Qiagen). The samples were then processed by the UCSF Functional Genomics Core as follows: after a quality control test, a single-end 50-bp RNA-Seq library (Ovation/NexteraXT kit, Illumina) was prepared, followed by sequencing on an Illumina HiSeq 4000 system. Sequencing provided 372 million total reads for the 6 samples, with an average of $81.9 \%$ of these reads aligning uniquely to the mouse genome (Ensembl Mouse GRCm38.78). Alignment was performed using the Splice-aware STAR aligner STAR_2.4.2a (63). Reads uniquely mapped to known mRNAs were used to identify genes with differential expression (FDR < 0.05) using the DESeq2 R package (64).

Zymograms. Lysates were prepared by homogenizing whole uterine tissue in RIPA buffer with complete protease inhibitors (Thermo Fisher Scientific) using Lysing Matrix D tubes (MP Biomedicals) and an MP Biomedicals homogenizer. For MMP-9 activity, $10 \mu \mathrm{g}$ protein from each sample was loaded onto gelatin-containing 10\% SDSPAGE gels (Novex zymograms, Life Technology). For elastase activity, we used homemade $12 \%$ gels containing $1 \mathrm{mg} / \mathrm{mL} \beta$-casein (SigmaAldrich). Following renaturation, enzyme activities were revealed by staining the gels with Brilliant Blue G (Sigma-Aldrich) followed by destaining. Image capture, analysis, and quantification were performed using the Chemidoc system (Bio-Rad) and the quantification tools of the ImageLab software (Bio-Rad). For each experiment, we normalized each inactive MMP-9 or elastase band intensity to its respective average intensity from the 3 or 4 samples run in each gel from mice housed under the normoxia condition.

Data availability. RNA-Seq data sets were deposited in the NCBI's Gene Expression Omnibus database (GEO GSE137431).

Statistics. Statistical analyses were performed in GraphPad Prism. $P$ values were determined using a 2-tailed Mann-Whitney $U$ test. In cases of multiple comparisons, the data were first analyzed by the Kruskal-Wallis test, and then Mann-Whitney-derived $P$ values were corrected using Bonferroni's adjustment. Statistical significance was defined as $P$ less than 0.05 .

Study approval. All animal experiments were approved by the Institutional Animal Care and Use Committees (IACUC) of UCSF and the NYU School of Medicine and were conducted in accordance with the relevant regulatory standards.

\section{Author contributions}

$\mathrm{KM}, \mathrm{AB}, \mathrm{SM}$, and $\mathrm{ACG}$ performed experiments. $\mathrm{KM}, \mathrm{AB}$, and $\mathrm{AE}$ designed experiments. CAL helped conceptualize the project. KM and $\mathrm{AE}$ analyzed the data and wrote the manuscript.

\section{Acknowledgments}

We thank E. Maltepe for discussions; N. Korets, D. McFall, P. Manandhar, and D. Rideaux for assistance with the histology; Z. Werb and F. DeMayo for mice; the UCSF Cytometry and Cell Sorting Core Facility (supported by DRC Center grant NIH P30 DK063720); and the UCSF Functional Genomics Core Facility. This study was supported by NIH grant R01 CA168755 (to AE).

Address correspondence to: Adrian Erlebacher, Department of Laboratory Medicine, UCSF, 513 Parnassus Avenue, Medical Sciences, S-1057B, San Francisco, California 94143-0451, USA. Phone: 415.502.3583; Email: adrian.erlebacher@ucsf.edu.
1. Coffelt SB, Wellenstein MD, de Visser KE. Neutrophils in cancer: neutral no more. Nat Rev Cancer. 2016;16(7):431-446.

2. Shaul ME, Fridlender ZG. Tumour-associated neutrophils in patients with cancer. Nat Rev Clin Oncol. 2019;16(10):601-620.

3. Vols S, Sionov RV, Granot Z. Always look on the bright side: anti-tumor functions of neutrophils. Curr Pharm Des. 2017;23(32):4862-4892.

4. Eruslanov EB, et al. Tumor-associated neutrophils stimulate $\mathrm{T}$ cell responses in earlystage human lung cancer. J Clin Invest. 2014;124(12):5466-5480.

5. Blaisdell A, et al. Neutrophils oppose uterine epithelial carcinogenesis via debridement of hypoxic tumor cells. Cancer Cell. 2015;28(6):785-799.

6. Kiss M, Van Gassen S, Movahedi K, Saeys Y, Laoui D. Myeloid cell heterogeneity in cancer: not a single cell alike. Cell Immunol.
2018;330:188-201.

7. Fridlender ZG, et al. Polarization of tumor-associated neutrophil phenotype by TGF-beta: "N1" versus “N2” TAN. Cancer Cell. 2009;16(3):183-194.

8. Engblom C, et al. Osteoblasts remotely supply lung tumors with cancer-promoting Siglec $F^{\text {high }}$ neutrophils. Science. 2017;358(6367): eaal5081.

9. Singhal S, et al. Origin and role of a subset of tumor-associated neutrophils with antigenpresenting cell features in early-stage human lung cancer. Cancer Cell. 2016;30(1):120-135.

10. Finisguerra V, et al. MET is required for the recruitment of anti-tumoural neutrophils. Nature. 2015;522(7556):349-353.

11. Mishalian I, Bayuh R, Levy L, Zolotarov L, Michaeli J, Fridlender ZG. Tumor-associated neutrophils (TAN) develop pro-tumorigenic properties during tumor progression. Cancer Immunol Immunother. 2013;62(11):1745-1756.
12. Liu $\mathrm{Z}$, et al. The serpin alpha1-proteinase inhibitor is a critical substrate for gelatinase $\mathrm{B} / \mathrm{MMP}-9$ in vivo. Cell. 2000;102(5):647-655.

13. Liu Z, Li N, Diaz LA, Shipley M, Senior RM, Werb Z. Synergy between a plasminogen cascade and MMP-9 in autoimmune disease. JClin Invest. 2005;115(4):879-887.

14. Houghton AM, et al. Neutrophil elastase-mediated degradation of IRS-1 accelerates lung tumor growth. Nat Med. 2010;16(2):219-223.

15. Kessenbrock K, Plaks V, Werb Z. Matrix metalloproteinases: regulators of the tumor microenvironment. Cell. 2010;141(1):52-67.

16. Bao S, Carr ED, Xu YH, Hunt NH. Gp91(phox) contributes to the development of experimental inflammatory bowel disease. Immunol Cell Biol. 2011;89(8):853-860.

17. Granot Z, Henke E, Comen EA, King TA, Norton L, Benezra R. Tumor entrained neutrophils 
inhibit seeding in the premetastatic lung. Cancer Cell. 2011;20(3):300-314.

18. Treffers LW, Hiemstra IH, Kuijpers TW, van den Berg TK, Matlung HL. Neutrophils in cancer. Immunol Rev. 2016;273(1):312-328.

19. Lodge KM, Thompson AA, Chilvers ER, Condliffe AM. Hypoxic regulation of neutrophil function and consequences for Staphylococcus aureus infection. Microbes Infect. 2017;19(3):166-176.

20. Lin N, Simon MC. Hypoxia-inducible factors: key regulators of myeloid cells during inflammation. JClin Invest. 2016;126(10):3661-3671.

21. Walmsley SR, et al. Hypoxia-induced neutrophil survival is mediated by HIF-1alphadependent NF-kappaB activity. JExp Med. 2005;201(1):105-115.

22. Hoenderdos K, et al. Hypoxia upregulates neutrophil degranulation and potential for tissue injury. Thorax. 2016;71(11):1030-1038.

23. Thompson AA, et al. Hypoxia-inducible factor $2 \alpha$ regulates key neutrophil functions in humans, mice, and zebrafish. Blood. 2014;123(3):366-376

24. Peyssonnaux C, et al. HIF-1alpha expression regulates the bactericidal capacity of phagocytes. J Clin Invest. 2005;115(7):1806-1815.

25. McGovern NN, et al. Hypoxia selectively inhibits respiratory burst activity and killing of Staphylococcus aureus in human neutrophils. JImmunol. 2011;186(1):453-463.

26. Corzo CA, et al. HIF-1 $\alpha$ regulates function and differentiation of myeloid-derived suppressor cells in the tumor microenvironment. JExp Med. 2010;207(11):2439-2453.

27. Marvel D, Gabrilovich DI. Myeloid-derived suppressor cells in the tumor microenvironment: expect the unexpected. JClin Invest. 2015;125(9):3356-3364.

28. Walmsley SR, et al. Prolyl hydroxylase 3 (PHD3) is essential for hypoxic regulation of neutrophilic inflammation in humans and mice. JClin Invest. 2011;121(3):1053-1063.

29. Cramer T, et al. HIF-1alpha is essential for myeloid cell-mediated inflammation. Cell. 2003;112(5):645-657.

30. Chouaib S, Noman MZ, Kosmatopoulos K, Curran MA. Hypoxic stress: obstacles and opportunities for innovative immunotherapy of cancer. Oncogene. 2017;36(4):439-445.

31. Dewhirst MW, Mowery YM, Mitchell JB, Cherukuri MK, Secomb TW. Rationale for hypoxia assessment and amelioration for precision therapy and immunotherapy studies. J Clin Invest. 2019;129(2):489-491.

32. Hatfield SM, et al. Immunological mechanisms of the antitumor effects of supplemental oxygenation. Sci Transl Med. 2015;7(277):277ra30.

33. Jayaprakash $P$, et al. Targeted hypoxia reduction restores $\mathrm{T}$ cell infiltration and sensitizes prostate cancer to immunotherapy. J Clin Invest. 2018;128(11):5137-5149.

34. Di Cristofano A, Ellenson LH. Endometrial carcinoma. Annu Rev Pathol. 2007;2:57-85.

35. Daikoku T, et al. Conditional loss of uterine Pten unfailingly and rapidly induces endometrial cancer in mice. Cancer Res. 2008;68(14):5619-5627.

36. Tibbles PM, Edelsberg JS. Hyperbaric-oxygen therapy. NEngl J Med.1996;334(25):1642-1648.

37. Maybin JA, Murray AA, Saunders PTK, Hirani N, Carmeliet P, Critchley HOD. Hypoxia and hypoxia inducible factor- $1 \alpha$ are required for normal endometrial repair during menstruation. Nat Commun. 2018;9(1):295.

38. Kallet RH, Matthay MA. Hyperoxic acute lung injury. Respir Care. 2013;58(1):123-141.

39. Kim MH, Yang D, Kim M, Kim SY, Kim D, Kang SJ. A late-lineage murine neutrophil precursor population exhibits dynamic changes during demand-adapted granulopoiesis. Sci Rep. 2017;7:39804

40. Campbell EL, et al. Transmigrating neutrophils shape the mucosal microenvironment through localized oxygen depletion to influence resolution of inflammation. Immunity. 2014;40(1):66-77.

41. Gross MW, Karbach U, Groebe K, Franko AJ, Mueller-Klieser W. Calibration of misonidazole labeling by simultaneous measurement of oxygen tension and labeling density in multicellular spheroids. Int J Cancer. 1995;61(4):567-573.

42. Jiang BH, Semenza GL, Bauer C, Marti HH. Hypoxia-inducible factor 1 levels vary exponentially over a physiologically relevant range of $\mathrm{O}_{2}$ tension. Am J Physiol. 1996;271(4 Pt 1):C1172-C1180.

43. Vandooren J, Van den Steen PE, Opdenakker G. Biochemistry and molecular biology of gelatinase B or matrix metalloproteinase-9 (MMP-9): the next decade. Crit Rev Biochem Mol Biol. 2013;48(3):222-272.

44. Borregaard N, Cowland JB. Granules of the human neutrophilic polymorphonuclear leukocyte. Blood.1997;89(10):3503-3521.

45. Shaul ME, et al. Tumor-associated neutrophils display a distinct N1 profile following TGF $\beta$ modulation: A transcriptomics analysis of pro- vs. antitumor TANs. Oncoimmunology. 2016;5(11):e1232221.

46. Condamine T, et al. Lectin-type oxidized LDL receptor-1 distinguishes population of human polymorphonuclear myeloid-derived suppressor cells in cancer patients. Sci Immunol. 2016;1(2):aaf8943.

47. Sundqvist M, et al. Elevated mitochondrial reactive oxygen species and cellular redox imbalance in human NADPH-oxidase-deficient phagocytes. Front Immunol. 2017;8:1828.

48. Hirahashi J, et al. Mac-1 signaling via Src-family and Syk kinases results in elastase-dependent thrombohemorrhagic vasculopathy. Immunity. 2006;25(2):271-283.

49. Clark RA, Klebanoff SJ. Neutrophil-mediated tumor cell cytotoxicity: role of the peroxidase system. J Exp Med.1975;141(6):1442-1447.

50. Riedle B, Kerjaschki D. Reactive oxygen species cause direct damage of Engelbreth-Holm-Swarm matrix. Am J Pathol. 1997;151(1):215-231.

51. Owen CA, Campbell EJ. The cell biology of leukocyte-mediated proteolysis. J Leukoc Biol. 1999;65(2):137-150.

52. Woods AA, Davies MJ. Fragmentation of extracellular matrix by hypochlorous acid. Biochem $\mathrm{J}$. 2003;376(Pt 1):219-227.

53. Metzler KD, Goosmann C, Lubojemska A, Zychlinsky A, Papayannopoulos V. A myeloperoxidase-containing complex regulates neutrophil elastase release and actin dynamics during NETosis. Cell Rep. 2014;8(3):883-896.

54. Geng S, et al. Emergence, origin, and function of neutrophil-dendritic cell hybrids in experimentally induced inflammatory lesions in mice. Blood.2013;121(10):1690-1700.

55. Hatfield SM, et al. Systemic oxygenation weakens the hypoxia and hypoxia inducible factor $1 \alpha$-dependent and extracellular adenosinemediated tumor protection. J Mol Med. 2014;92(12):1283-1292.

56 . Wang X, Chen D. Purinergic regulation of neutrophil function. Front Immunol. 2018;9:399.

57. Petrova V, Annicchiarico-Petruzzelli M, Melino G, Amelio I. The hypoxic tumour microenvironment. Oncogenesis. 2018;7(1):10.

58. Soyal SM, et al. Cre-mediated recombination in cell lineages that express the progesterone receptor. Genesis. 2005;41(2):58-66.

59. $\mathrm{Vu} \mathrm{TH}$, et al. MMP-9/gelatinase B is a key regulator of growth plate angiogenesis and apoptosis of hypertrophic chondrocytes. Cell. 1998;93(3):411-422.

60. Collins MK, Tay CS, Erlebacher A. Dendritic cell entrapment within the pregnant uterus inhibits immune surveillance of the maternal/fetal interface in mice. JClin Invest. 2009;119(7):2062-2073.

61. Blaisdell A, Erlebacher A. Immunofluorescence and Immunohistochemistry. In: Croy BA, Yamada AT, DeMayo FJ, Adamson SL, eds. The Guide to Investigation of Mouse Pregnancy. Boston, Massachusetts, USA: Elsevier; 2014:577-589.

62. Weibel ER. Stereological principles for morphometry in electron microscopic cytology. Int Rev Cytol. 1969;26:235-302.

63. Dobin A, et al. STAR: ultrafast universal RNA-seq aligner. Bioinformatics. 2013;29(1):15-21.

64. Love MI, Huber W, Anders S. Moderated estimation of fold change and dispersion for RNA-seq data with DESeq2. Genome Biol. 2014;15(12):550. 